biete der Festkörperphysik erwarten. Voraussetzung ist allerdings, daß zunächst der Emissionsmechanismus noch näher geklärt wird.

Hinsichtlich der mit dieser Untersuchungsmethode $\mathrm{zu}$ behandelnden Probleme sei hier nur auf einige wenige Punkte hingewiesen, die von besonderem Interesse sein dürften. Da ist zunächst die Möglichkeit zu nennen, auf diese Weise den Oberflächenzustand eines Festkörpers zu untersuchen. Man darf nach dem oben Gesagten wohl hoffen, daß diese Methode die Energieverteilung der Fehlstellen, und zwar speziell die an oder in der Nähe der Oberfläche, liefern kann. Denn in einem vorgegebenen Kristall entspricht ja eine bestimmte Aktivierungsenergie $\varepsilon$ auch einer bestimmten Fehlstellenart. Die Zuordnung bestimmter Gitterfehler zu diesen Aktivierungsenergien wird zwar oft Paralleluntersuchungen nach anderen Methoden, insbesondere Leitfähigkeits- und Lumineszenzuntersuchungen, erforderlich machen; die besondere Bedeutung dieser Methode liegt aber darin, daß man nun auch Kenntnis über die Verteilung dieser Fehlstellen in der Nähe der Oberfläche erhält. Dies dürfte für die verschiedensten Gebiete auch von erheblichem praktischem Interesse sein. Hier seien nur 2 Beispiele genannt: die heterogene Katalyse, bei der die Elektronenbindung

40 D. A. D o w d e n, J. Chem. Soc. 1950, 242-265.

$41 \mathrm{~K}$. H a uffe, R. G l a n g u. H. J. Engell, Z. phys. Chem. 201, 223-245 [1952]. an der Oberfläche des Katalysators eine wesentliche Rolle spielt ${ }^{40,41}$, und das Gebiet der Pulvermetallurgie, wo z. B. die sog. Sinteraktivität entscheidend von der Struktur der Oberfläche abhängt. - Auch für die Halbleiterforschung dürfte diese Methode von Interesse sein. - Ferner sollte man mit der Methode die Stabilität bestimmter Fehlstellen bei und nach den verschiedensten Vorbehandlungen untersuchen können (vgl. Abschnitt III). Auf die Möglichkeit der Untersuchungen an Metalloberflächen ${ }^{42}$ sowie bei Zerkleinerungsvorgängen ${ }^{43}$ sei nur hingewiesen.

Gegenüber der Methode der Leitfähigkeitsmessung bestehen hier die Vorteile, daß keine Störungen durch Ionenleitung und bei Pulvern keine Kontaktschwierigkeiten auftreten, sowie, daß wegen der geringen Ströme Störungen durch Raumladungen wesentlich unwahrscheinlicher sind. Im Gegensatz zu Phosphoreszenzuntersuchungen ergibt sich die Möglichkeit, auch die große Zahl der nicht phosphoreszierenden Stoffe zu untersuchen.

Ich danke Herrn Dr. K. S i g w a r t für den ersten Hinweis auf diese Untersuchungsmethodik und die Anregung zu ihrer Bearbeitung; Herrn $\mathrm{H}$ a r e $\mathrm{n} \mathrm{d} \mathrm{z} \mathrm{a} \mathrm{bin} \mathrm{ich} \mathrm{für} \mathrm{die} \mathrm{Aus-}$ führung der numerischen Rechnungen zu Dank verpflichtet. Für wertvolle Anregungen und Diskussionen gebührt mein besonderer Dank Herrn Prof. J. J a u m a n n, Köln.

42 J. K r a m e r, Metalloberfläche 4 A, 1-6 [1955].

43 J. K r a m e r, Ber. dtsch. Keram. Ges. 30, 204 [1953].

\title{
Über die Grenzen der Meßbarkeit kleiner Temperaturdifferenzen und Wärmemengen, dargestellt an einer Theorie der Thermonadel
}

\author{
Von R. Dahlberg* \\ (Z. Naturforschg. 10 a, 953-970 [1955] ; eingegangen am 28. September 1955)
}

\begin{abstract}
Nach einer kurzen Zusammenstellung von Verfahren, die die Herstellung von Thermoelementen mit geringer Wärmekapazität und -ableitung ermöglichen, werden Konstruktion und thermische Daten der vom Verfasser entwickelten mikroskopisch feinen „Thermonadeln“ wiedergegeben. Um die meßtechnischen Möglichkeiten dieser Thermonadeln beurteilen zu können, wird zunächst eine einfache Theorie des Thermoelementes als Wärmekraftmaschine entwickelt: Der Nutzeffekt wird definiert als der Quotient aus der durch Peltier- und Thomson-Effekt erzeugten elektrischen Energie und den durch die an der Meßstelle des Thermoelementes reversibel und irreversibel aufgenommenen Wärme-

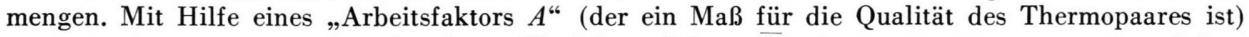
wird ein theoretischer und praktischer "Gütefaktor $G$ bzw. $\bar{G}^{“}$ als das Verhältnis des Nutzeffektes eines Thermoelementes zum Nutzeffekt einer Carnot-Maschine definiert. Dieses Verhältnis gibt an, um welchen Faktor der Nutzeffekt eines Thermoelementes „theoretisch“ und „praktisch“ hinter dem thermodynamisch größtmöglichen Nutzeffekt zurückbleibt. Aus der Tatsache, daß $G$ niemals größer als 1 werden kann, wird eine obere Grenze für die Arbeitsfaktoren $A \leqq 1$ und damit (in Abhängigkeit
\end{abstract}

* Karlsruhe (Baden), Speyererstr. $14 \mathrm{~b}$. 
von den spez. elektrischen und spez. Wärmeleitfähigkeiten beliebiger Thermopaare) auch eine obere Grenze für die diff. Thermokräfte postuliert.

Unter Beachtung der natürlichen Grenze, die die thermischen Schwankungserscheinungen jeder elektrischen Messung setzen, wird danach für jeden speziellen Fall mit Hilfe des Nutzeffektes eines Thermoelementes eine Grenze der Meßmöglichkeit für kleine Temperaturdifferenzen und Wärmemengen abgeleitet. Es wird gefolgert, daß das Produkt aus einer gemessenen Temperaturdifferenz $T_{1}-T_{0}$ und der von einem Thermoelement für die Messung von $T_{1}-T_{0}$ verbrauchten Wärmemenge $Q$ einen kleinsten und in jedem speziellen Fall verschiedenen Wert nicht unterschreiten kann.

Unter idealen Grenzbedingungen läßt sich aus diesem Produkt ein Maß für die Genauigkeit ableiten, mit der in einem thermodynamischen System die Temperatur und der Wärmeinhalt überhaupt angebbar sind. Für praktische Messungen werden danach die kleinsten Temperaturdifferenzen berechnet, die mit Hilfe von Thermoelementen unter speziellen Voraussetzungen noch meßbar sind, und in einer Tabelle werden die in diesem Zusammenhange interessierenden Materialdaten für einige Thermopaare zusammengestellt.

Abschließend wird die thermische Beeinflussung kleiner Meßobjekte durch den Meßvorgang diskutiert.

\section{Problemstellung}

$\mathrm{B}$ ei physikalischen, chemischen und vor allem bei biologischen Untersuchungen ist es oftmals interessant, neben der mittleren Temperatur eines betrachteten Systems auch die Temperaturverhältnisse in seinen räumlichen Teilen zu kennen. Besonders dann, wenn das System Wärmequellen enthält (etwa bei inhomogener Energieabsorption oder beim Ablauf ortsabhängiger chemischer Prozesse), kann die Kenntnis der räumlichen Temperaturverteilung wichtig sein, weil sie Rückschlüsse auf die Einzelheiten des Energieumsatzes erlaubt. Häufig bereitet aber in der Praxis die Messung von Temperaturspitzen, die auf relativ kleine - etwa nur unter der Lupe oder dem Mikroskop gut sichtbare - räumliche Bereiche beschränkt sind, erhebliche Schwierigkeiten. Der Grund hierfür liegt darin, daß jedes Thermometer für die Messung Wärme verbraucht, die es dem Meßobjekt entzieht. Dadurch wird die zu messende Temperatur mehr oder minder stark verändert. Diese Störung kann bei sehr kleinen Meßobjekten unzulässig werden und wird nur dadurch verringert, daß man für die Messung Thermometer mit hinreichend kleinem Wärmeverbrauch benutzt.

Diese Notwendigkeit tritt aber nicht nur bei der Messung von zeitlich praktisch konstanten Temperaturdifferenzen in kleinen Objekten auf, sondern ebenso bei der Registrierung sehr schneller Temperaturschwankungen wie sie $z$. B. bei adiabatischen Kompressionen und Dilatationen vorkommen. Hier sind - unabhängig von der Größe des Meßobjektes - die aufgenommenen Wärmemengen infolge der Kürze der zur Verfügung stehenden Meßzeiten so gering, daß ebenfalls nur Thermometer mit hinreichend kleiner Wärmekapazität zur Mes-

1 F. L i e n e w e g, „Temperaturmessung“, S. 182, Leipzig 1950, Akad. Verlagsges. sung geeignet sind. Von der Größe der Wärmekapazität hängt es (neben dem Wärmeübergang) ab, ob und mit welcher Amplitude und Phase das Thermometer den Temperaturschwankungen folgen kann $^{1}$. Je kleiner die Wärmekapazität ist, um so schneller wird die Meßstelle eine neue Temperatur annehmen können, um so geringer ist die thermische Trägheit des Thermometers.

Von allen gebräuchlichen Thermometerkonstruktionen läßt sich am einfachsten das Thermoelement so ausführen, daß es als Thermometer kleiner Wärmekapazität verwendbar ist. Es besteht im Prinzip aus zwei an den Enden miteinander elektrisch leitend verbundenen Drähten verschiedenen Materials. Eine Temperaturdifferenz zwischen den beiden Verbindungsstellen erzeugt über die thermoelektrische Spannung und den Ohmschen Widerstand im Drahtkreis einen Strom, der ein $\mathrm{Ma} ß$ für die bestehende Temperaturdifferenz ist. Da die thermoelektrische Spannung und der Strom in erster Näherung unabhängig von dem Temperaturverlauf im Drahtkreis und nur abhängig von der Temperaturdifferenz zwischen den Verbindungsstellen sind, bestimmt deren Masse im wesentlichen die Wärmekapazität des Thermoelementes. (Praktisch von Bedeutung ist nur die Wärmekapazität der einen Verbindungsstelle, die die "Meßstelle“ ist, weil die andere ohnehin als „Vergleichsstelle“ auf konstanter Temperatur bleibt.) Macht man die Masse der Meßstelle immer kleiner, wobei man verständlicherweise auch die Durchmesser der verwendeten Drähte immer kleiner wählen muß, dann kann man theoretisch sehr einfach Thermometer mit äußerst kleiner Wärmekapazität herstellen. In der Praxis kommt man jedoch bei der Konstruktion von Thermoelementen aus dünnen Drähten bald an eine Grenze des Brauchbaren und Möglichen, die vor allem in der Schwierigkeit der Handhabung dünner Drähte ihren Grund 
hat. Wenn jedoch bei spezieller Verwendung - z. B. als Strahlungsmeßgerät - keine gefährliche mechanische Beanspruchung des fertigen Thermoelementes mehr auftritt, dann bestimmt im wesentlichen das Herstellungsverfahren der Meßstelle diese Grenze.

\section{A) Thermoelemente geringer Wärmekapazität und -ableitung}

\section{Zusammenstellung einiger Herstellungsverfahren}

Zur Herstellung von Thermoelementen mit Meßstellen möglichst kleiner Masse sind in der Literatur eine ganze Reihe zum Teil sehr sinnreicher Verfahren und Kunstgriffe angegeben worden. So beschreiben $\mathrm{Pf} \mathrm{un}{ }^{2}$ und $\mathrm{M}$ e y e ${ }^{3}$ Lötvorrichtungen, mit denen Meßstellen aus Weichlot geringster Masse erzielt werden können, und $\mathrm{Kant}$ or owi c z und $\mathrm{R}$ e i n e c k e ${ }^{4}$ schlagen vor, kleine Stückchen Schwammgold (durch Festklopfen) als Lötmasse zu verwenden. Obwohl diese Verfahren noch relativ grob erscheinen, lassen sich mit ihrer Hilfe doch schon Meßstellen mit Wärmekapazitäten von $10^{-5}$ bis $10^{-6} \mathrm{cal} /{ }^{\circ}$ bei einiger Übung herstellen.

Wesentlich mehr leistet eine Schweißung von dünnen Drähten mit Hilfe von Kondensatorentladungen, wie sie $\mathrm{Z}$ d r a le $\mathrm{k}$ und $\mathrm{Wr}$ a n g a ${ }^{5}$ beschrieben haben. Nach diesem Verfahren konnten vom Verfasser Drähte mit Durchmessern bis zu 13,5 $\mu$ (unter Luftabschluß im Paraffinbad) verschweißt werden. Die Wärmekapazitäten der Meßstellen betrugen hier nur noch $10^{-8}$ bis $10^{-9} \mathrm{cal} /{ }^{\circ}$.

Ein besonders originelles Verfahren zur Herstellung von dünnen Thermoelementen wurde von $\mathrm{M}$ üll $\mathrm{e} \mathrm{r}^{7}$ angegeben. Ein dünner Draht (oder auch eine dünne Folie) wird galvanisch auf seiner halben Länge mit einer Schicht eines zweiten Metalles überzogen. Mit Hilfe einer nachfolgenden thermischen Diffusion wird die überzogene Hälfte des Drahtes in eine Legierung verwandelt, und die Utbergangsstelle von legiertem zu unlegiertem Draht wirkt als Meßstelle des fertigen Thermoelementes. Müller konnte nach diesem Verfahren, das Verwendung beim Bau empfindlicher Strahlungsmeßgeräte fand, auch Thermoelemente aus Drähten mit Durchmessern bis zu $3 \mu$ herstellen. Nimmt man für die Größe der Meßstelle dieses Thermoelementes ein Drahtstückchen von der Länge des Durchmessers an der Übergangsstelle vom legierten zum unlegierten Draht an, dann beträgt die Wärmekapazität der Meßstelle hier nur noch $10^{-10}$ bis $10^{-11} \mathrm{cal} /{ }^{\circ}$.

Ein ähnliches, sehr sinnreiches Verfahren zur Herstellung

2 A. H. P f u n d, Phys. Z. 13, 870 [1912].

3 Fr. M e y e r, Z. Fernmeldetechnik 1, 13 [1920].

4 O. Kantorowicz u. R. Reinecke, Z. techn. Phys. 11, 547 [1930].

5 O. Z d ra lek u. I. W r a n g a, Elektrotech. Z. 56, 579,991 [1935].

6 R. D a h l b e r g, Diplomarbeit, Frankfurt a. M. 1951.

7 C. M ü 11 e r, Naturwiss. 19, 416 [1931] sowie D. R.P. Nr. 554315 , Klasse 21 b, Gruppe 27 (10.6. 1926).

${ }^{8}$ H. W ils o n u. T. D. E p p s, Proc. Phys. Soc., Lond. 32, 326 [1920]. von Thermoelementen haben Wils on und Epp s ${ }^{8}$ sowie später $\mathrm{Kohaut}^{9}$ und $\mathrm{Jones}^{10}$ angegeben. Es besteht darin, daß - ebenso wie bei dem Verfahren von Müller die halbe Länge eines Drahtes mit einem Metallmantel eines zweiten Metalles umgeben wird, ohne daß danach eine Legierungsbildung erfolgt. Der Übergang von der normalen Drahthälfte zum Manteldraht wirkt auch hier als Meßstelle eines Thermoelementes ${ }^{11}$. Der Verfasser ${ }^{6}$ konnte nach diesem Verfahren Thermoelemente aus Wollastondrähten mit Durchmessern bis zu $3 \mu$ herstellen.

Trurnit ${ }^{12}$ gelang sogar die Herstellung von „Thermosonden" aus Drähten bis zu 1,5 $\mu$ Durchmesser. Er überzog dazu einen Draht sehr dünn mit Bakelitlack, schnitt die „Spitze“ des isolierten Drahtes ab und stellte eine leitende Verbindung zwischen dieser Schnittfläche und den Zuleitungen der Thermosonde über die Lackschicht durch Aufdampfen von Silber her. Die Wärmekapazität der feinsten Thermosonden dürfte nur noch etwa $10^{-11}$ bis $10^{-12} \mathrm{cal} /{ }^{\circ}$ betragen haben.

Bei allen diesen Thermoelementkonstruktionen zeigt sich die oben erwähnte Schwierigkeit der Handhabung und Anwendung der fertigen Thermoelemente als Thermometer. Für praktische Temperaturmessungen sind nur die robusteren und unempfindlicheren Ausführungsformen verwendbar, weil bei jeder Messung die Meßstelle des Thermoelementes in Wärmekontakt mit dem Meßobjekt gebracht werden muß, was u. U. eine erhebliche mechanische Beanspruchung bedeutet. Alle aus Drähten mit Durchmessern unter $20 \mu$ hergegestellten Thermoelemente sind wegen ihrer mechanischen Empfindlichkeit dafür praktisch ungeeignet. Selbst die von Trurnit entwickelten Thermosonden konnten für biologische Temperaturmessungen nur mit Drahtdurchmessern über $15 \mu$ verwendet werden. Die untere Grenze der Wärmekapazität von Drahtthermoeleṃenten, bei deren Verwendung die experimentellen Schwierigkeiten noch erträglich bleiben, liegt etwa zwischen $10^{-8}$ bis $10^{-9} \mathrm{cal} /{ }^{\circ}$. Diese Größe reicht für sehr viele praktische Temperaturmessungen in dem eingangs erwähnten Sinne auch vollkommen aus, und viele wichtige physiologische Untersuchungen, z. B. die Wärmemessungen von $\mathrm{Hill} \mathrm{l}^{13}$ am quergestreiften Muskel, sind mit Thermoelementen größerer thermischer Trägheit (Drahtdurchmesser etwa $50 \mu$ ) durchgeführt worden.

Es gibt aber daneben einige recht interessante Fragestellungen, bei denen man entweder noch wesentlich feinere Thermometer benötigen würde (z. B. energetische Probleme der experimentellen Zellforschung) oder bei denen man neben großer Feinheit des Thermometers eine höhere mechanische Festigkeit verlangt, als sie Drahtthermoelemente besitzen (z. B. Messung thermischer Effekte in Ultraschallfeldern).

9 A. K o h a u t, Wiener Ber. 137 (2a), 436-467 [1928] sowie Wiener Anz. 17, 207 [1928].

10 R. V. J o n e s, J. Sci. Instrum. 14, 83 [1937].

11 L. G e il ing, Brevet d'invention, Gr. 12 - C., 3, No 1.020.139, Imprimerie Nationale, Paris (15e). Die von L. G e i l i n g beschriebenen "Schichtelemente“ (Multimetallblock) sind eine originelle, komplexe Abart dieser Thermoelementkonstruktion.

${ }_{12}$ H. J. T r u rnit, Naturwiss. 27, 805 [1939].

13 A. V. H i l l, Physiol. Rev. 2, 310 [1922]. 


\section{Konstruktion und Eigenschaften der Thermonadel}

Als experimenteller Ausweg bei derartigen Fragestellungen wurde vom Verfasser ${ }^{14}$ ein Thermoelement in Form einer Nadel entwickelt, das bei geringster Wärmekapazität eine relativ sehr hohe mechanische Festigkeit hat ${ }^{14}$ a

Die Konstruktion dieser sogenannten „Thermonadel" beruht darauf, daß man die in der biologischen Technik der Zelloperationen gebräuchliche Spitznadel aus Glas oder Quarz durch Bedampfen im Hochvakuum mit zwei thermoelektrisch günstigen Metallen I und II in ein Thermoelement "verwandelt“, bei dem die Spitznadel den Träger für die Aufdampfschichten abgibt und dem Thermoelement seine eigene, relativ sehr hohe mechanische Festigkeit verleiht. Das konstruktive Problem der Thermonadel besteht lediglich darin, die beiden Metallschichten so an der Trägernadel entlang zu führen, daß sie nur an der äußersten Spitze der Nadel (etwa an der Vorderfläche der Spitze) elektrisch leitend verbunden sind und sonst entlang der ganzen Nadel isoliert gegeneinander verlaufen. Die Verbindungsstelle der beiden Metallschichten I und II an der äußersten Nadelspitze ist die Meßstelle der Thermonadel, während die Vergleichsstellen die Kontakte der Zuleitungsdrähte sind, die man an den dickeren Teil der Nadel auf die beiden Aufdampfschichten I und II anlegen oder mit Indium als Lötmasse anlöten kann. Abb. 1 zeigt eine Thermonadel im Längsschnitt.

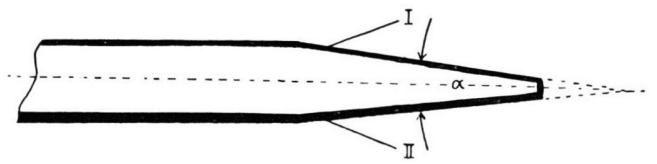

Abb. 1. Bedampfte Glasnadel im Längsschnitt.

Eine Isolation der beiden Metallschichten entlang der Nadel kann man dadurch erreichen, daß man zunächst die Metallschichten I und II auf die Unterseite und Oberseite der Nadel (um $180^{\circ}$ verdreht) so aufdampft, daß eine Schichtdickenverteilung entsteht, die vom Winkel $\varphi$ der Flächennormalen der Oberfläche abhängt. Abb. 2 zeigt die Schichtdickenverteilung im Querschnitt.

Danach kann man mit Hilfe einer chemischen, thermischen oder elektrochemischen Behandlung

14 R. D a h 1 b e r g, Strahlentherapie 94, 300 [1954].

14a Die Anregung zur Entwicklung von Thermoelementen mit extrem kleiner Wärmekapazität und -ableitung erhielt der

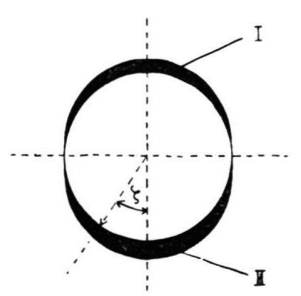

Abb. 2. Bedampfte Glasnadel im Querschnitt mit Schichtdickenverteilung.

einen Teil einer oder auch beider Metallschichten in eine nichtleitende oder auch flüchtige $(z$. B. bei $\mathrm{Fe}$ und $\mathrm{Ni}$ durch Behandlung mit CO) Metallverbindung I' und II" überführen. Dadurch verringern sich die leitenden Querschnitte der Aufdampfschichten, sie rücken auseinander, und die eventuell gebildeten nichtleitenden Schichten verstärken noch die dadurch erreichte elektrische Isolation. Abb. 3 gibt den Aufbau der Thermonadel im Querschnitt wieder.

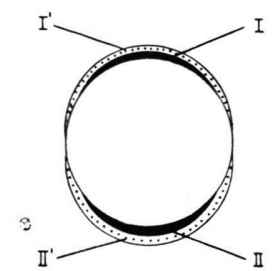

Abb. 3. Thermonadel im Querschnitt.

Verwendet man etwa für die Metallschichten sogenannte „Ventilmetalle“ (Ta, Nb, Zr, Al, Bi, Sb u. a.), dann kann man einen Teil der Schicht dadurch in die entsprechenden isolierenden Metalloxyde verwandeln, daß man die bedampfte Nadel als Anode in einen geeigneten Elektrolyten bringt. (Für Al und Bi z. B. eine 3-proz. Ammoniumzitratlösung.) Da die Dicke der durch den Formierungsprozeß gebildeten Oxydschichten der angelegten Spannung in erster Näherung proportional sind, kann man durch geeignete Dicke der Aufdampfschichten eine saubere Isolation entlang der ganzen Nadel erreichen, ohne daß die leitende Brücke an ihrer äußersten Spitze unterbrochen wird. In ganz analoger Weise können auch chemische oder thermische Verfahren zur Isolation verwendet werden.

Die Abb. $1^{*}$ bis $4^{*}$ zeigen zwei Thermonadeln, von denen einzelne mit Spitzendurchmessern bis zu etwa $0,1 \mu$ hergestellt werden konnten. Die Mikro-

Verfasser von Herrn Prof. Dr. B. R a j ew sk y, Frankfurt am Main.

* Abb. 1* bis 4* auf Tafel S. 956 a. 
R. Dahlberg, Über die Grenzen der Meßbarkeit kleiner Temperaturdifferenzen und Wärmemengen, dargestellt an einer Theorie der Thermonadel (S. 953).

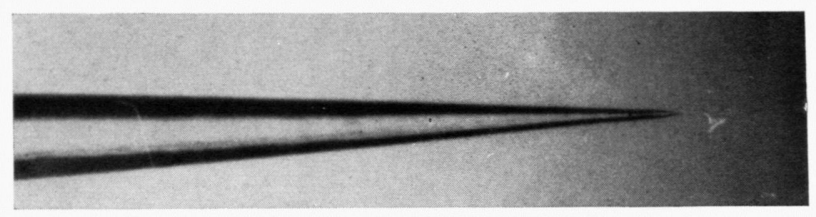

Abb. 1*. Thermonadel mit durchoxydierten Seitenzonen. Vergr. 475-fach $(1 \mathrm{~mm} \sim 2 \mu)$.

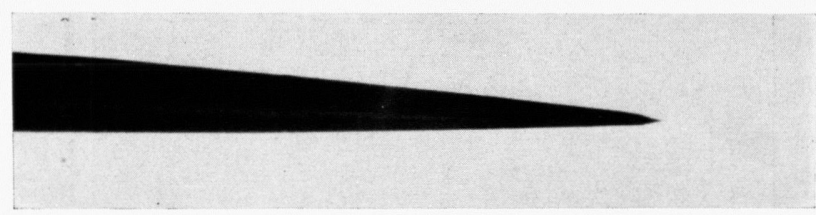

Abb. $2^{*}$. Gleiche Nadel wie Abb. $1^{*}$, um $90^{\circ}$ gedreht. Vergr. 475-fach $(1 \mathrm{~mm} \sim 2 \mu)$.

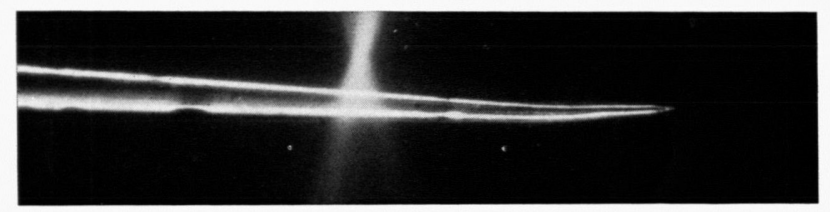

Abb. ${ }^{*}$. Thermonadel mit durchoxydierten Seitenzonen. Vergr. 145-fach $(1 \mathrm{~mm} \sim 7 \mu)$.

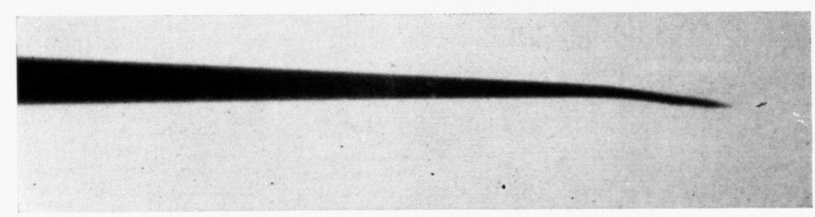

Abb. $4^{*}$. Gleiche Nadel wie Abb. $3^{*}$, um $90^{\circ}$ gedreht. Vergr. 145-fach $(1 \mathrm{~mm} \sim 7 \mu)$. 
A. Bardocz und F. Varsanyi, Erzeugung zeitlich aufgelöster Spektren von Hochfrequenz-Funkenentladungen kleiner Energie (S. 1031).

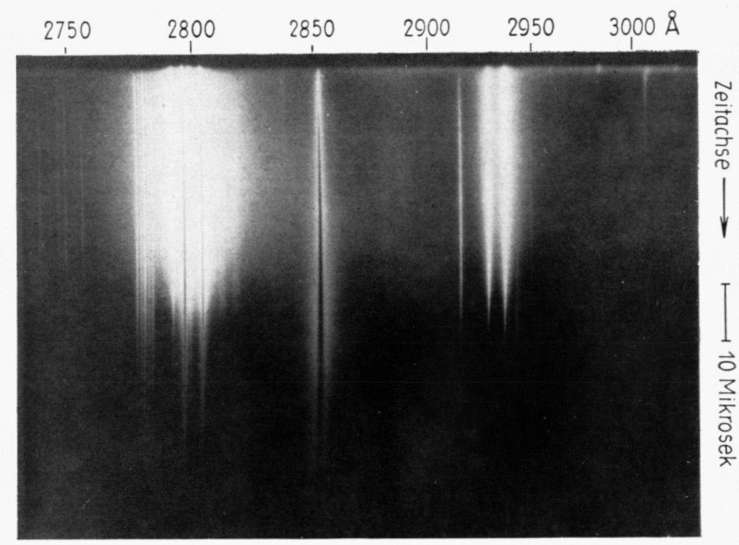

Abb. 2. Zeitlich aufgelöstes Spektrum von Magnesium zwischen den Wellenlängen $2750-3000 \AA$.

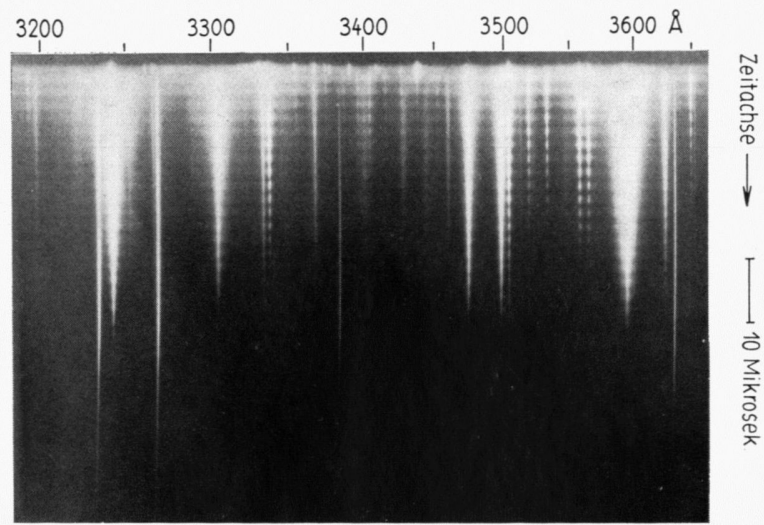

Abb. 3. Zeitlich aufgelöstes Spektrum von Antimon zwischen den Wellenlängen $3200-3650$ Å. 
photos machen die Trennung der beiden Aufdampfschichten sichtbar: die durchoxydierten Seitenzonen sind lichtdurchlässig geworden und erscheinen im Bild als heller Streifen (Abb. $1^{*}$ und $3{ }^{*}$ ), der nach Drehung um $90^{\circ}$ verschwunden ist (Abb. $2^{*}$ und $\left.4{ }^{*}\right)$.

Insgesamt wurden vom Verfasser bisher Thermonadeln mit folgenden Thermopaaren hergestellt:

$$
\begin{array}{ll}
\mathrm{Bi}-\mathrm{Sb} & \mathrm{Fe}-\mathrm{Ni} \\
\mathrm{Bi}-\mathrm{Cu} & \mathrm{Fe}-\mathrm{Konstantan} \\
\mathrm{Bi}-\mathrm{Te} & \mathrm{Cr}-\mathrm{Ni} \\
\mathrm{Bi}-\mathrm{Ge} & \mathrm{Cr}-\mathrm{Pd} \\
\mathrm{Sb}-\mathrm{Ni} & \mathrm{Au}-\mathrm{Pd} \\
\mathrm{Sb}-\mathrm{Al} &
\end{array}
$$

Die differentiellen Thermokräfte dieser Thermonadeln lagen stets unter den Werten für die Metalle im kompakten Zustand und waren vor allem für die Halbleiter und die diesen näher stehenden Metalle ( $\mathrm{Bi}$ und $\mathrm{Sb}$ ) im Bereich zwischen 0,5 bis $0,05 \mu$ Schichtdicke stark schichtdickenabhängig. (Für Thermonadeln $\mathrm{Bi}-\mathrm{Sb}$ mit Aufdampfschichten von 100 bzw. $50 \mathrm{~m} \mu$ betrug die differentielle Thermokraft bei $1 \mu$ Spitzendurchmesser im Durchschnitt nur noch etwa $30 \mu \mathrm{V} /{ }^{\circ}$.)

Die vor der Verwendung noch im Hochvakuum mit $\mathrm{SiO}$ bzw. $\mathrm{ThF}_{4}$ rotationssymmetrisch schutzbedampften Thermonadeln erweisen sich als Thermometer mit sehr geringer Wärmekapazität und einer hohen mechanischen Festigkeit. Es ist mit ihnen z. B. gelungen, Temperaturmessungen an schwingenden Gasbläschen (Kavitationsbläschen) im Ultraschallfeld durchzuführen ${ }^{15,15 a}$.

Abb. 4 gibt die berechnete Wärmekapazität $C_{\mathrm{T}}$ der Thermonadel in Abhängigkeit vom Spitzendurchmesser $d$ wieder. Es ist dabei vorausgesetzt, daß nur die Masse der leitenden Brücke an der äußersten Nadelspitze (50-m $\mu$-Schicht Eisen) die Meßstelle der Thermonadel bildet. Zum Vergleich ist die Wärmekapazität $C_{\mathrm{D}}$ der Meßstellen von Drahtthermoelementen aus $\mathrm{Fe}-\mathrm{Ni}$-Drähten mit Drahtdurchmesern gleich den Spitzendurchmessern $d$ der Thermonadeln eingezeichnet. Es ist hierbei angenommen, daß die Meßstelle des Drahtthermoelemen-

15 R. P a p e u. R. D a h l b e r g, Max-Planck-Institut für Biophysik, Frankfurt a. M. (noch unveröffentlicht).

15a An Resonanzbläschen von $10 \mu$ Durchmesser - Schallfrequenz $1 \mathrm{MHz}$ - konnten Temperaturspitzen von $90^{\circ} \mathrm{C}$ maximal in Meßzeiten bis zu $100 \mu$ sec registriert werden. Die gewonnenen Meßwerte stimmen gut mit theoretischen Uberlegungen von $\mathrm{S} \mathrm{ch}$ ä $\mathrm{f}$ e $\mathrm{r}$ und $\mathrm{S} \mathrm{ch}$ w a ${ }^{16}$ überein. tes die Masse eines Drahtstückchens der Länge $d$ besitzt.

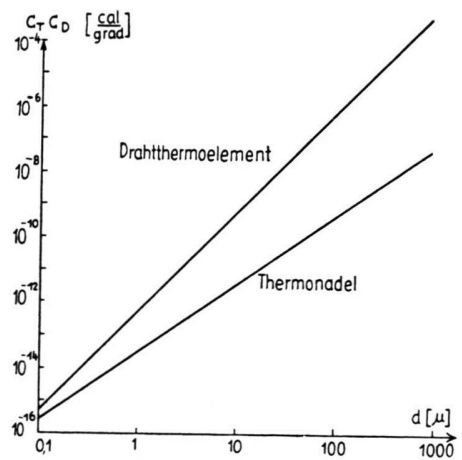

Abb. 4. Wärmekapazität $C_{\mathrm{T}}$ von Thermonadeln und Wärmekapazität $C_{\mathrm{D}}$ von Drahtthermoelementen in Abhängigkeit vom Spitzendurchmesser $d$ (gleich Drahtdurchmesser) der Thermonadeln.

Abb. 5 gibt eine Vorstellung von der thermischen Trägheit der Thermonadel, wie sie sich aus der Lösung der nichtstationären Wärmeleitungsgleichung unter vereinfachten Annahmen ergibt ${ }^{17}$. Es sind die Materialdaten von Eisen in kompaktem Zustand dabei verwendet. Man sieht aus Abb. 5, daß für den idealen Grenzfall eines ungestörten Wärmeüberganges eine Eisenschicht bis zu einer Tiefe von $D=0,1 \mu$ bereits nach $10^{-6} \mathrm{~s}$ einer plötzlichen Temperaturerhöhung $T_{1}-T_{0}$ (bei $D=0$ ) zu $99 \%$ gefolgt ist.

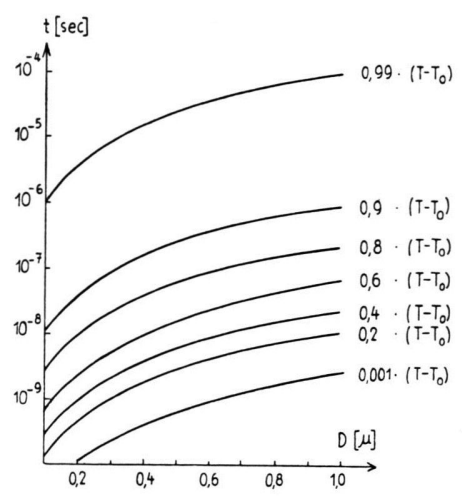

Abb. 5. Geschwindigkeitsdiagramm des Eindringens einer Wärmewelle in einen Eisenzylinder zur Veranschaulichung der thermischen Trägheit einer $\mathrm{Fe}-\mathrm{Ni}$-Thermonadel. In der Beschriftung der Kurven muß jeweils $T_{1}$ statt $T$ stehen.

16 H. S c häf e r u. H. S c hw a n, Ann. Phys., Paris 43, 99 [1943].

${ }^{17}$ Die Thermonadel wurde als Eisenzylinder idealisiert (die Glasspitze nicht berücksichtigt) und eine Wärmeströmung in radialer Richtung vernachlässigt, was wegen der Beschränkung auf sehr schnelle Temperaturänderungen möglich ist ${ }^{17} \mathrm{a}$.

17a J a k o b, Handb. Physik 11, 69 [1926]. 
Für den stationären Zustand läßt sich der Wärmewiderstand $W_{\mathrm{T}}$ der Thermonadel berechnen. Vernachlässigt man auch hier eine Wärmeabgabe „nach außen “, dann ist der Wärmewiderstand relativ hoch, obwohl die Trägernadel aus Glas eine zusätzliche Wärmeableitung bedeutet.

Abb. 6 gibt den stationären Wärmewiderstand in Abhängigkeit vom Spitzendurchmesser $d$ der Thermonadel wieder, wobei angenommen wurde, daß der Wärmewiderstand nur auf einer Länge von $100 d$ wirksam ist ${ }^{17 \mathrm{~b}}$, und daß der Öffnungswinkel $\alpha$ der kegelförmigen Nadelspitze $10^{\circ}$ beträgt. Die maximale Schichtdicke $D_{\mathrm{m}}$ ist als Parameter angegeben. Als Vergleich sind die Wärmewiderstände von $\mathrm{Fe}-\mathrm{Ni}$-Drahtthermoelementen - gleichfalls in einer Länge von $100 d$ unter den gleichen Bedingungen eingezeichnet, wobei die Drahtdurchmesser wieder gleich den Spitzendurchmessern der Thermonadeln sind.

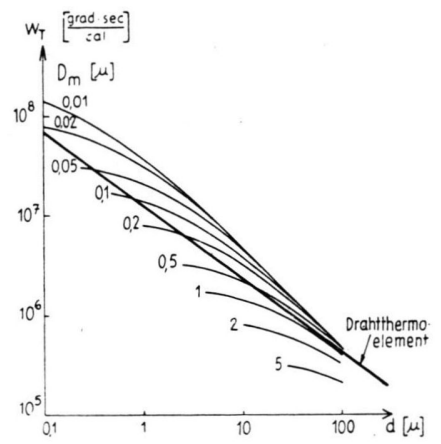

Abb. 6. Wärmewiderstand $W_{\mathrm{T}}$ der Thermonadel in Abhängigkeit von $d$ mit der maximalen Schichtdicke $D_{\mathrm{m}}$ als Parameter.

Abb. 7 stellt das Verhältnis der Wärmewiderstände $W / W_{\mathrm{n}}$ als Funktion von $d$ mit dem Öffnungswinkel $\alpha$ als Parameter dar. Die maximale Schichtdicke ist dabei $D_{\mathrm{m}}=5 \cdot 10^{-6} \mathrm{~cm}$ angenommen.

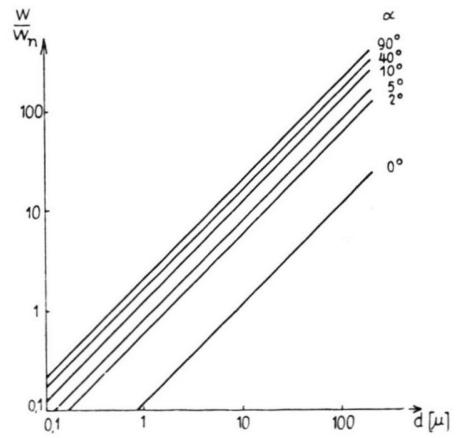

Abb. 7. Verhältnis des Wärmewiderstandes $W$ der beiden Metallschichten zum Wärmewiderstand $W_{n}$ der Trägernadel in Abhängigkeit von $d$ mit dem Öffnungswinkel $\alpha$ der Kegelspitze als Parameter. $D_{\mathrm{m}}=0,05 \mu \mathrm{Fe}-\mathrm{Ni}$.
Der geringe Wärmeverbrauch bei relativ sehr hoher mechanischer Festigkeit sowie die große Handlichkeit selbst bei größter Feinheit sichern der Thermonadel eine Reihe von Anwendungsmöglichkeiten. Sie kann nicht nur als Strahlungsmeßgerät geringster thermischer Trägheit (man kann z. B. die Temperatur einer sehr dünnen Folie, die die Wärmestrahlung absorbiert, mit ihr messen) Verwendung finden, sondern sie wird vor allem überall dort, wo es darauf ankommt, eventuell sehr kurzzeitige Temperaturschwankungen oder Temperaturgradienten in relativ kleinen Raumgebieten oder Objekten zu erfassen, die bisher schon bestehenden meßtechnischen Möglichkeiten erweitern. Bei kugelsymmetrischen Wärmeleitungsvorgängen, bei denen also eine „punktförmige“ Wärmequelle vorhanden ist, wird sie auch als Mikrokalorimeter (über die Messung radialer Temperaturgradienten) verwendet werden können, was vor allem für biologische Fragestellungen wichtig werden könnte.

\section{B) Theoretische Überlegungen}

\section{Das Thermoelement als Wärmekraftm a s chine}

Zur Beurteilung der Thermonadel als Meßgerät ist es zweckmäßig, nach der Grenze ihrer meßtechnischen Möglichkeiten zu fragen. Daß eine solche Begrenzung existieren muß, ergibt sich aus der Tatsache, daß die Thermonadel aufgenommene Wärmeenergie in elektrische Energie umwandelt, die mit einem Meßinstrument gemessen wird. Wie jedes Thermoelement ist auch die Thermonadel eine Wärmekraftmaschine, die diese Umwandlung nur mit einem bestimmten Nutzeffekt bewirkt, der höchstens so groß sein kann, wie der Nutzeffekt einer Carnot-Maschine. Da der Messung sehr kleiner elektrischer Energien durch die thermischen Schwankungserscheinungen (Brownsche Molekularbewegung, Johnson-Rauschen u. a.) eine natürliche Grenze gesetzt wird, muß auch die Messung sehr kleiner Wärmemengen zum Beispiel mit einem Thermoelement unter gegebenen Bedingungen eine Grenze besitzen, die wesentlich vom Nutzeffekt der Umwandlung der Wärmemenge in elektrische Energie abhängt. Da die während einer Meßzeit aufgenommene Wärme-

17b Die zu messende Temperaturdifferenz $T_{1}-T_{0}$ soll in diesem Abstand von der Meßstelle auf 5\% ihres Wertes abgesunken sein. 
menge aber unter anderem auch von der bestehenden Temperaturdifferenz abhängt, wird gezeigt werden können, daß auch für die Messung von kleinen Temperaturdifferenzen diese natürliche Begrenzung existiert.

Den Nutzeffekt eines Thermoelementes kann man nach der Thomsonschen Theorie der Thermoelektrizität ${ }^{18}$ definieren als das Verhältnis der durch die thermoelektrischen Effekte (Peltier- und ThomsonEffekt) erzeugten elektrischen Leistung zu den vom Thermoelement pro Zeiteinheit reversibel und irreversibel aufgenommenen Wärmemengen.

Nach der ersten Thomsonschen Gleichung ist die im Thermoelement erzeugte elektrische Leistung:

$$
\boldsymbol{U} \boldsymbol{I}=\left[\pi_{1}-\pi_{0}+\int_{0}^{1}\left(\sigma_{\mathrm{II}}-\sigma_{\mathrm{I}}\right) \mathrm{d} T\right] I .
$$

Dabei bedeuten: $\quad U=\int_{T_{0}}^{T_{1}} \varepsilon_{\mathrm{I}, \mathrm{II}} \mathrm{d} T \quad$ die integrale

Thermospannung, $\varepsilon_{\text {I, II }}$ die differentielle Thermospannung, $I$ der Thermostrom, $\pi_{1} I$ die an der Meßstelle aufgenommene Peltier-Wärme, $\pi_{0} I$ die an der Vergleichsstelle abgegebene Peltier-Wärme, sowie $\sigma_{\mathrm{I}}$ und $\sigma_{I I}$ die Thomson-Koeffizienten der Schenkelmaterialien I und II des Thermoelementes.

Der Nutzeffekt des Thermoelementes läßt sich damit schreiben:

$$
\eta=U I /\left(\pi_{1} I+j\right)
$$

$\tau_{1} I$ im Nenner von Gl. (2) ist die an der Meßstelle reversibel pro Zeiteinheit aufgenommene (Peltier-) Wärme, und $j$ ist die an der Meßstelle pro Zeiteinheit irreversibel aufgenommene Wärme. Die Differenz der Thomson-Wärmen

$$
I \int_{0}^{1}\left(\sigma_{\mathrm{II}}-\sigma_{\mathrm{I}}\right) \mathrm{d} T
$$

in den beiden Schenkeln des Thermoelementes erscheint nicht explizit im Nenner von Gl. (2), weil die Thomson-Wärmen sich über das gesamte Temperaturgefälle in beiden Schenkeln I und II verteilen. Durch sie wird jedoch die Größe der Wärmemenge mitbestimmt, die an der Meßstelle (infolge der Wärmeleitung) irreversibel aufgenommen wird. Die Thomson-Wärmen bewirken im allgemeinen wegen ihres verschiedenen Vorzeichens eine Asymmetrierung des Temperaturverlaufs in den beiden Schenkeln des Thermoelementes und müssen damit

18 W. M e i s s n e r, Handb. d. Exp. Phys. Bd. 11, 2. Teil, 397, Leipzig 1935. implizit (ebenso wie die Joulesche Wärme) in einem noch abzuleitenden Ausdruck für die Wärmemenge $j$ enthalten sein, die an der Meßstelle pro Zeiteinheit irreversibel aufgenommen wird.

Zur Berechnung von $j$ sollen folgende Annahmen gemacht werden:

1. Die Wärmeabgabe in die „äußere Umgebung“ des Thermoelementes soll vernachlässigbar klein sein.

2. In dem betrachteten Temperaturintervall $T_{1}-T_{0}$ ( $T_{1}$ absolute Temperatur der Meßstelle, $T_{0}$ absolute Temperatur der Vergleichsstelle) sollen die spez. elektrischen Widerstände $\varrho_{\mathrm{I}}$ und $\varrho_{\mathrm{II}}$ sowie die spez. Wärmeleitfähigkeiten $\lambda_{\mathrm{I}}$ und $\lambda_{\text {II }}$ der Schenkelmaterialien hinreichend konstant sein.

3. Die beiden Schenkel I und II seien gleichlang und ihre Querschnitte $f_{\mathrm{I}}$ und $f_{\mathrm{II}}$ seien auf der ganzen Länge $l$ der Schenkel konstant. (Drahtthermoelement.)

Die Temperaturverteilungen $T_{\mathrm{I}}(x)$ und $T_{\mathrm{II}}(x)$ als Funktionen der Längenkoordinate $x \quad\left(x=0\right.$ bei $T_{0}$, $\mathrm{x}=l$ bei $T_{1}$ ) in den beiden Schenkeln des Thermoelementes ergeben sich dann durch Integration der beiden Gleichungen:

$$
\begin{gathered}
\lambda_{\mathrm{I}} f_{\mathrm{I}} \frac{\mathrm{d}^{2} T_{\mathrm{I}}}{\mathrm{d} x^{2}}+\sigma_{\mathrm{I}} I \frac{\mathrm{d} T_{\mathrm{I}}}{\mathrm{d} x}+\frac{I^{2} \varrho_{\mathrm{I}}}{f_{\mathrm{I}}}=0, \\
\lambda_{\mathrm{II}} f_{\mathrm{II}} \frac{\mathrm{d}^{2} T_{\mathrm{II}}}{\mathrm{d} x^{2}}-\sigma_{\mathrm{II}} I \frac{\mathrm{d} T_{\mathrm{II}}}{\mathrm{d} x}+\frac{f_{\mathrm{II}}}{I^{2} \varrho_{\mathrm{II}}}=0 .
\end{gathered}
$$

Man kann eine für unsere Zwecke brauchbare Näherungslösung dieser Differentialgleichungen gewinnen, wenn man für $\mathrm{d} T / \mathrm{d} x$ in Gl. (3) den Ausdruck einsetzt, der sich aus der Lösung der Gl. (3) ergibt, wenn vorher $\sigma=0$ gesetzt wird. Dann lassen sich für die Wärmemengen $j_{\mathrm{I}}$ und $j_{\mathrm{II}}$, die an der Meßstelle vom Schenkel I und II irreversibel aufgenommen werden, die Beziehungen gewinnen:

$$
\begin{aligned}
j_{\mathrm{I}}=\lambda_{\mathrm{I}} f_{\mathrm{I}}\left[\frac{l^{2}}{12} \frac{I^{3} \sigma_{\mathrm{I}} \varrho_{\mathrm{I}}}{f_{\mathrm{I}}^{3} \lambda_{\mathrm{I}^{2}}}\right. & \\
& \left.-\frac{l}{2} \frac{I^{2} \varrho_{\mathrm{I}}}{f_{\mathrm{I}}{ }^{2} \lambda_{\mathrm{I}}}-\frac{T_{1}-T_{0}}{2} \frac{I \sigma_{\mathrm{I}}}{f_{\mathrm{I}} \lambda_{\mathrm{I}}}+\frac{T_{1}-T_{0}}{l}\right], \\
j_{\mathrm{II}}=\lambda_{\mathrm{II}} f_{\mathrm{II}}[ & -\frac{l^{2}}{12} \frac{I^{3} \sigma_{\mathrm{II}} \varrho_{\mathrm{II}}}{f_{\mathrm{II}}{ }^{3} \lambda_{\mathrm{II}}{ }^{2}} \\
& \left.-\frac{l}{2} \frac{I^{2} \varrho_{\mathrm{II}}}{f_{\mathrm{II}} \lambda_{\mathrm{II}}}+\frac{T_{1}-T_{0}}{2} \frac{I \sigma_{\mathrm{II}}}{f_{\mathrm{II}} \lambda_{\mathrm{II}}}+\frac{T_{1}-T_{0}}{l}\right] .
\end{aligned}
$$

Setzt man in Gl. (4) die Abkürzungen $\varrho_{\mathrm{I}} l / f_{\mathrm{I}}=R_{\mathrm{I}}$ Ohmscher Widerstand des Schenkels I, $l / f_{\mathrm{I}} \lambda_{\mathrm{I}}=W_{\mathrm{I}}$ Wärmewiderstand des Schenkels I, sowie die ent- 
sprechenden Abkürzungen für den Schenkel II und ferner

$$
R_{\mathrm{I}}+R_{\mathrm{II}}=R, 1 /\left(1 / W_{\mathrm{I}}+1 / W_{\mathrm{II}}\right)=W,
$$

dann kann man für die gesamte irreversibel aufgenommene Wärme schreiben:

$$
j=j_{\mathrm{I}}+j_{\mathrm{II}}=\frac{T_{1}-T_{0}}{W}-\frac{1}{2} I^{2} R+\frac{1}{2} I\left(\sigma_{\mathrm{II}}-\sigma_{\mathrm{I}}\right)\left(T_{1}-T_{0}\right)-\frac{1}{1 \overline{2}} I^{3}\left(\sigma_{\mathrm{II}} R_{\mathrm{II}} W_{\mathrm{II}}-\sigma_{\mathrm{I}} R_{\mathrm{I}} W_{\mathrm{I}}\right) .
$$

Dies ist in Gl. (2) einzutragen.

Man kann nun sehr einfach zeigen, daß es bei jedem Thermoelement möglich ist, durch die Erfüllung einer bestimmten konstruktiven Bedingung die beiden rechten Terme in Gl. (6) zum Verschwinden zu bringen. Dadurch würde der Nutzeffekt offenbar ein Maximum. In diesem Falle müßte gelten:

$$
\frac{1}{2} I\left(\sigma_{\mathrm{II}}-\sigma_{\mathrm{I}}\right)\left(T_{1}-T_{0}\right)=\frac{1}{12} I^{3}\left(\sigma_{\mathrm{II}} R_{\mathrm{II}} W_{\mathrm{II}}-\sigma_{\mathrm{I}} R_{\mathrm{I}} W_{\mathrm{I}}\right)
$$

oder nach einigen Umformungen:

$$
\frac{\sigma_{\mathrm{II}}}{\sigma_{\mathrm{I}}}=\frac{6\left(T_{1}-T_{0}\right)-I^{2} R_{\mathrm{I}} W_{\mathrm{I}}}{6\left(T_{1}-T_{0}\right)-I^{2} R_{\mathrm{II}} W_{\mathrm{II}}} .
$$

Man sieht sofort, daß $\sigma_{\mathrm{II}} / \sigma_{\mathrm{I}}=1$, also $\sigma_{\mathrm{II}}=\sigma_{\mathrm{I}}$ wird, wenn

$$
R_{\mathrm{I}} W_{\mathrm{I}}=R_{\mathrm{II}} W_{\mathrm{II}} \quad \text { oder } \quad R_{\mathrm{I}} / R_{\mathrm{II}}=W_{\mathrm{II}} / W_{\mathrm{I}}
$$

wird. Das bedeutet, daß die Differenz der ThomsonWärmen Null wird, wenn sich die elektrischen Widerstände der beiden Schenkel des Thermoelementes umgekehrt verhalten wie die entsprechenden Wärmewiderstände. Unter Einhaltung der durch Gl. (9) bestimmten Bedingung kann man für den Nutzeffekt des Thermoelementes nun schreiben, s. Anm. * :

$$
\eta=\frac{U I}{\left(\pi_{1} I+\frac{T_{1}-T_{0}}{W}-\frac{1}{2} I^{2} R\right)} .
$$

Da gemäß Gl. (1) auch der Einfluß des ThomsonEffektes auf die integrale Thermospannung $U$ verschwunden ist, bleibt bei Konstanz von $\varrho_{\text {I, II }}$ und $\lambda_{\text {I, II }}$ im betrachteten Temperaturintervall auch die

* Man sieht, daß das Thermoelement offenbar eine CarnotMaschine wird, wenn der Wärmestrom $\left(T_{1}-T_{0}\right) / W$ (bei Quellenfreiheit) gleich wird der halben Jouleschen Wärme, die im (kurzgeschlossenen) Thermoelement erzeugt wird. Dann ist

$$
\eta=\frac{U I}{\pi_{1} I}=\frac{U}{\pi_{1}}=\frac{T_{1}-T_{0}}{T_{1}} .
$$

Weiter unten wird gezeigt werden, daß dieser ideale Grenzfall jedoch selbst mit Hilfe eines ,idealen Thermopaares" nur mit $T_{1}-T_{0} \rightarrow T_{1}$ angenähert werden könnte. differentielle Thermospannung $\varepsilon$ konstant. Man kann deshalb schreiben:

$$
U I=I^{2} R=\frac{1}{R} \varepsilon^{2}\left(T_{1}-T_{0}\right)^{2} .
$$

Aus Gl. (10) wird damit, wenn man außerdem noch die jetzt streng geltende Thomsonsche Beziehung

einsetzt:

$$
\pi_{1}=\varepsilon T_{1}
$$

$$
\eta=\varepsilon^{2}\left(T_{1}-T_{0}\right) /\left[\varepsilon^{2} T_{1}+R / W-\frac{1}{2} \varepsilon^{2}\left(T_{1}-T_{0}\right)\right] .
$$

Diese Gleichung läßt sich mit Hilfe des Gesetzes von Wiedemann-Franz und Lorenz noch weiter vereinfachen.

Für die sog. Lorenz-Zahl $L_{\mathrm{I}}$ des Materials vom Schenkel I gilt:

$$
L_{\mathrm{I}}=\frac{\lambda_{\mathrm{I}} \varrho_{\mathrm{I}}}{T}=\frac{1}{T} \frac{\varrho_{\mathrm{I}} l / f_{\mathrm{I}}}{l / f_{\mathrm{I}} \lambda_{\mathrm{I}}}=\frac{1}{T} \frac{R_{\mathrm{I}}}{W_{\mathrm{I}}} .
$$

Wählt man für $T$ die mittlere absolute Temperatur $\left(T_{1}+T_{0}\right) / 2$ im Temperaturintervall $T_{1}-T_{0}$, dann kann man für die Wärmewiderstände der beiden Schenkel schreiben:

$$
W_{\mathrm{I}}=\frac{R_{\mathrm{I}}}{L_{\mathrm{I}}} \frac{2}{T_{1}+T_{0}}, \quad W_{\mathrm{II}}=\frac{R_{\mathrm{II}}}{L_{\mathrm{II}}} \frac{2}{T_{1}+T_{0}} .
$$

Der Gesamtwärmewiderstand des Thermoelementes ist dann:

$$
W=\frac{1}{\left(\mathrm{l} / W_{\mathrm{I}}+\mathrm{l} / W_{\mathrm{II}}\right)}=\frac{R_{\mathrm{I}} R_{\mathrm{II}}}{R_{\mathrm{II}} L_{\mathrm{I}}+L_{\mathrm{II}} R_{\mathrm{I}}} \frac{2}{T_{1}+T_{0}} .
$$

Das Verhältnis von elektrischem zum Wärmewiderstand wird damit:

$$
\frac{R}{W}=\frac{\left(R_{\mathrm{I}}+R_{\mathrm{II}}\right)\left(R_{\mathrm{II}} L_{\mathrm{I}}+L_{\mathrm{II}} R_{\mathrm{I}}\right)}{R_{\mathrm{I}} R_{\mathrm{II}}} \frac{T_{1}+T_{0}}{2} .
$$

Setzt man

$$
R_{\mathrm{I}}=n R_{\mathrm{II}} \quad \text { und } \quad L_{\mathrm{I}}=p L_{\mathrm{II}},
$$

dann wird

$$
R / W=\left[L_{\mathrm{I}}(1+\mathrm{l} / n)+L_{\mathrm{II}}(1+n)\right] \cdot\left(T_{1}+T_{0}\right) / 2
$$


ein Minimum, wenn

$$
n=\sqrt{p}, \quad \text { also } \quad R_{\mathrm{I}} / R_{\mathrm{II}}=\sqrt{L_{\mathrm{I}} / L_{\mathrm{II}}}
$$

ist. Man erkennt mit Hilfe der Definition der LorenzZahl - Gl. (14) - sofort, daß dies nichts anderes als die in Gl. (9) abgeleitete Bedingung für den maximalen Nutzeffekt des Thermoelementes ist. Das Verhältnis $R / W$ wird unter dieser Bedingung ein Minimum, für das man schreiben kann:

$$
\begin{aligned}
\left(\frac{R}{W}\right)_{\min } & =L_{\mathrm{I}}\left(1+\frac{1}{\sqrt{L_{\mathrm{I}} / L_{\mathrm{II}}}}\right)^{2} \frac{T_{1}+T_{0}}{2} \\
& =L_{\mathrm{II}}\left(\sqrt{\frac{L_{\mathrm{I}}}{L_{\mathrm{II}}}}+1\right)^{2} \frac{T_{1}+T_{0}}{2} .
\end{aligned}
$$

Setzt man nun noch zur Abkürzung

$$
\frac{\varepsilon^{2}}{L_{\mathrm{II}}\left(\sqrt{L_{\mathrm{I}} / L_{\mathrm{II}}}+1\right)^{2}}=A,
$$

dann kann man für den Nutzeffekt eines Thermoelementes unter den vorausgesetzten optimalen konstruktiven Bedingungen schreiben:

$$
\eta=\frac{A}{A+1} \frac{2\left(T_{1}-T_{0}\right)}{T_{1}+T_{0}} .
$$

Dieser Nutzeffekt $\eta$ eines Thermoelementes kann niemals größer werden als der Nutzeffekt $\left(T_{1}-T_{0}\right) / T_{1}$ einer Carnot-Maschine. Wir wollen das Verhältnis des Nutzeffektes $\eta$ eines Thermoelementes zum größtmöglichen Nutzeffekt einer Carnot-Maschine als den "theoretischen Gütefaktor $G^{\text {“ }}$ des Thermoelementes einführen, für den also gilt:

$$
\eta T_{1} /\left(T_{1}-T_{0}\right)=G \text { mit } 0 \leqq G \leqq 1
$$

oder mit Berücksichtigung von Gl. (23) :

$$
G=\frac{A}{A+1} \frac{2 T_{1}}{T_{1}+T_{0}} .
$$

$G$ erreicht offenbar seinen größten Wert, wenn $T_{1} /\left(T_{1}+T_{0}\right) \rightarrow 1$ geht. Daraus muß gefolgt werden, $\mathrm{da} ß A$ niemals einen größeren Wert als 1 annehmen kann, weil sonst $G>1$ werden würde, was ja unmöglich ist. Nimmt man einmal an, daß

$$
A=\varepsilon^{2} / L_{\mathrm{II}}(\sqrt{p}+1)^{2}
$$

von der absoluten Temperatur unabhängig ist - eine Annahme, deren Berechtigungsumfang noch geprüft werden müßte - , dann läßt sich aus diesem Grenzübergang auch eine obere Begrenzung für die dif-

19 J. K o l l e r t, Elektrotechn. Z. 11, 333 [1890].

20 E. A lt e n kirch, Phys. Z. 10, 560 [1909].

21 M. T el ke s, J. Appl. Phys. 18, 1116 [1947]. ferentiellen Thermospannungen $\varepsilon_{\max }$ ableiten, die man höchstenfalls bei Thermopaaren mit beliebigen Lorenz-Zahlen erwarten darf. Abb. 8 gibt diese obere Grenze der differentiellen Thermospannungen wieder. Man sieht, daß bei Thermopaaren, die etwa das Wiedemann-Franzsche Gesetz erfüllen $\left(L=2 \cdot 10^{-8}\right.$ bis $4 \cdot 10^{-8} \mathrm{~V}^{2} /^{\circ}$ ) keine höheren differentiellen Thermospannungen bei $T_{1}=300^{\circ} \mathrm{K}$ als etwa 300 bis $400 \mu \mathrm{V} /{ }^{\circ}$ auftreten können. Für kleine Temperaturdifferenzen $T_{1} /\left(T_{1}+T_{0}\right) \rightarrow 1 / 2$ wird der Gütefaktor $G$ von der Temperatur unabhängig und $\mathrm{Gl}$. (25) geht über in

$$
G=A /(A+1) \leqq 1 / 2 .
$$

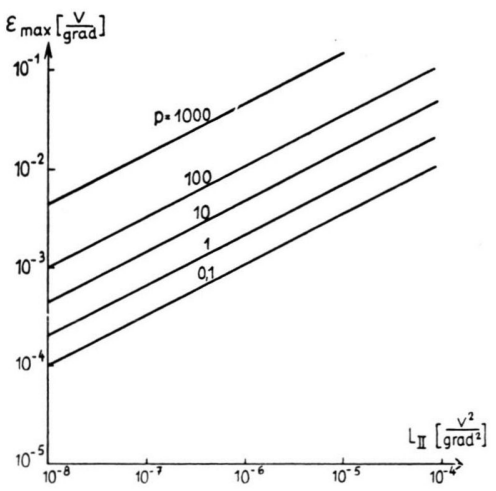

Abb. 8. Theoretisch postulierte Höchstwerte für die differentiellen Thermospannungen $\varepsilon_{\max }$ zwischen zwei Thermomaterialien mit den Lorenz-Zahlen $L_{\mathrm{I}}$ und $L_{\mathrm{II}}$ mit $p=L_{\mathrm{I}} / L_{\mathrm{II}}$ als Parameter.

Diese Beziehung (26) hätten wir auch ohne weiteres aus Gl. (2) gewinnen können, wenn wir uns von vorneherein bei der Definition des Nutzeffektes auf kleine Temperaturdifferenzen beschränkt und den Thomson-Effekt sowie die Joulesche Wärme vernachlässigt hätten, wie dies Lord $\mathrm{R}$ a y l e i g h ${ }^{23}, \mathrm{Kol}$. le $\mathrm{rt}^{19}$, A l tenkirch ${ }^{20}$, Telkes ${ }^{21}, \mathrm{Ge}$ il ing ${ }^{22}$ u. a. getan haben. Der Nutzeffekt wäre dann einfach gewesen:

$$
\eta=\frac{I^{2} R}{\pi_{1} I+\left(T_{1}-T_{0}\right) / W}=\frac{\varepsilon^{2}\left(T_{1}-T_{0}\right)}{\varepsilon^{2} T_{1}+R / W} .
$$

Abb. 9 gibt den theoretischen Gütefaktor $G$ für kleine Temperaturdifferenzen in Abhängigkeit von $A$ wieder.

Die Größe $A \leqq 1$ hat im Falle kleiner Temperaturdifferenzen eine sehr einfache, anschauliche Bedeu-

${ }^{22}$ L. G eiling, Bull. schweiz. elektrotechn. Ver. 41, 536 [1950]. 


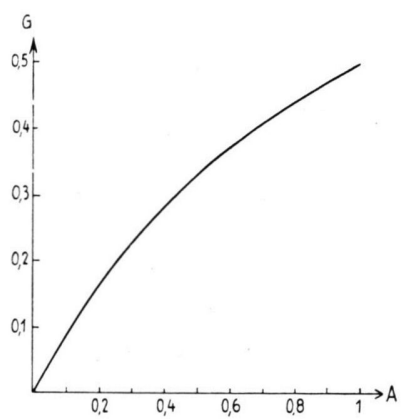

Abb. 9. Theoretischer Gütefaktor $G$ als Funktion des Arbeitsfaktors $A$ für kleine Temperaturdifferenzen.

tung. Sie ist das Verhältnis der an der Meßstelle pro Zeiteinheit reversibel aufgenommenen Wärme $\pi_{1} I$ zur irreversibel (durch Leitung) aufgenommenen Wärme $\left(T_{1}-T_{0}\right) / W$, und zwar im Falle der günstigsten Konstruktion

$$
A=\frac{\pi_{1} I}{\left(T_{1}-T_{0}\right) / W}=\frac{\varepsilon^{2} T_{1}\left(T_{1}-T_{0}\right)}{\left(T_{1}-T_{0}\right) R / W}=\frac{\varepsilon^{2}}{L_{\mathrm{II}}\left(\sqrt{L_{\mathrm{I}} / L_{\mathrm{II}}}+1\right)^{2}} .
$$

Die Größe $A$ wird nur von den Materialeigenschaften des Thermopaares bestimmt und stellt für energetische Betrachtungen eine brauchbarere Charakterisierung des Thermopaares dar als die differentielle Thermokraft. Wir wollen $A$ den "Arbeitsfaktor" eines Thermopaares nennen, weil er für kleine Temperaturdifferenzen, auf die wir uns im folgenden beschränken werden, ein Maß für die Arbeitsfähigkeit der aufgenommenen Wärme gibt ${ }^{24}$.

Wenn man nun danach fragt, mit welchem Nutzeffekt ein Thermoelement Wärme in praktisch auch entnehmbare elektrische Energie umwandelt, dann sieht man bald, daß der Gütefaktor $G$ nur einen praktisch unerreichbaren Grenzwert darstellt. Um den nach ihm errechenbaren Nutzeffekt zu erzielen, muß das Thermoelement die ideale konstruktive Form haben, was u. a. bedeutet, daß man die Elementschenkel (in denen der Temperaturgradient nirgends Null ist) kurzschließen muß. Obwohl es prinzipiell auch möglich sein müßte, wenigstens einen Teil der elektrischen Leistung $I^{2} R$ in den vom Wärmestrom durchflossenen Schenkeln zu gewinnen (man könnte ja den Thermostrom zerhacken und das kurzgeschlossene Thermoelement als Primärspule eines Transformators ausbilden), wird man in der Praxis im allgemeinen wohl doch einen Ver-

23 Lord J. W. R a y le i g h, Phil. Mag. 20, 361 [1885].

${ }^{24} \mathrm{~L}$. G e i l i n g ${ }^{24 a}$ verwendet den ähnlich gebauten Aus- braucher (außerhalb des Temperaturgefälles) in den thermoelektrischen Kreis legen, was einen zusätzlichen Widerstand bedeutet. Außerdem kann auch der Wärmestrom in der Praxis größer sein als $j=\left(T_{1}-T_{0}\right) / W$ (etwa infolge von Wärmeabgabe der Elementschenkel an die Umgebung oder z. B. bei der Thermonadel durch die Wärmeleitung der Trägernadel aus Glas oder Quarz). Wird dadurch der minimale Wärmestrom $j=\left(T_{1}-T_{0}\right) / W$ um den Faktor

$$
\begin{aligned}
& F=1+W / W_{\mathrm{n}} \geqq 1, W_{\mathrm{n}}= \underset{\text { Wärmewiderstand }}{ } \\
& \text { der Trägernadel, }
\end{aligned}
$$

der vom Aufbau des Thermoelementes abhängt und „Formfaktor“ genannt werden soll, vergrößert, und besitzt etwa der eingeschaltete Verbraucher den elektrischen Widerstand

$$
R_{\mathrm{a}}=a R, \quad \text { also } \quad R_{\mathrm{a}}+R=R(a+1),
$$

dann kann man einen praktischen Nutzeffekt $\bar{\eta}$ definieren:

$$
\bar{\eta}=\frac{\frac{\varepsilon^{2}\left(T_{1}-T_{0}\right)^{2}}{R(a+1)} \frac{a}{a+1}}{\pi_{1} \frac{\varepsilon\left(T_{1}-T_{0}\right)}{R(a+1)}+j F}
$$

und ganz analog zum „theoretischen Gütefaktor $G^{\text {“ }}$ daraus einen praktischen Gütefaktor $\bar{G}$ ableiten:

$$
\bar{G}=\frac{\varepsilon^{2}}{\varepsilon^{2}+\left[L_{\mathrm{I}}(1+1 / n)+L_{\mathrm{II}}(1+n)\right](a+1) F} \frac{a}{a+1} .
$$

Abb. 10 zeigt $\bar{G}$ in Abhängigkeit von $a$ für ein Thermoelement mit $A=0,25, F=2$ und $n^{2}=p$.

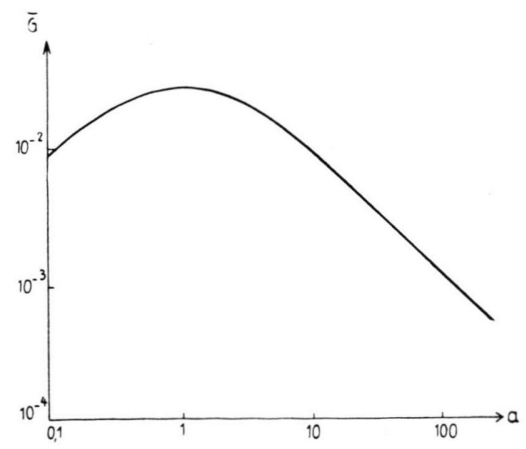

Abb. 10. Praktischer Gütefaktor $\bar{G}$ eines Thermoelementes mit dem Arbeitsfaktor $A=0,25$ in Abhängigkeit von $\dot{a}=R_{\mathrm{a}} / R$ für $F=2$ und $n^{2}=p$.

druck $\varepsilon^{2} /\left(L_{\mathrm{I}}+L_{\mathrm{II}}\right)$, den er „Thermometallzahl“ nennt, zur Charakterisierung von Thermopaaren.

24a L. G e il ing, Z. angew. Phys. 3, 467 [1951]. 
Der praktische Gütefaktor $\bar{G}$ wird ein Maximum für $a=\sqrt{(A+F) / F}, F=1$ und $n=\sqrt{L_{\mathrm{I}} / L_{\mathrm{II}}}=\sqrt{p}$. In diesem Falle ist der Verbraucher "angepaßt" und die Elementkonstruktion optimal. Dann ist (für beliebige Arten von Thermoelementen) :

$$
\bar{G}_{\max }=\frac{A}{A+1+\sqrt{A+1}} \frac{\sqrt{A+1}}{1+\sqrt{A+1}}=\frac{\sqrt{A+1}-1}{\sqrt{A+1}+1} .
$$

Abb. 11 gibt $\bar{G}_{\max }$ in Abhängigkeit von $A$ wieder.

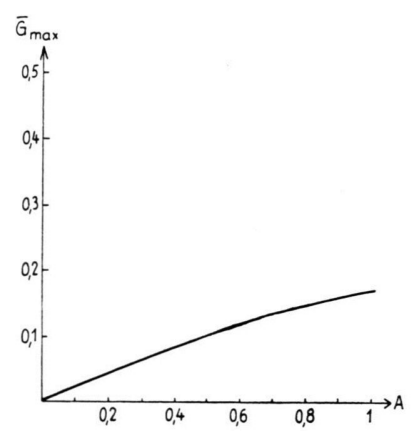

Abb. 11. Maximaler praktischer Gütefaktor $\bar{G}_{\max }$ als Funktion des Arbeitsfaktors $A$ für kleine Temperaturdifferenzen.

Ließe man ein bekanntes Thermopaar mit relativ hohem Arbeitsfaktor etwa 90\% $\mathrm{Bi}-10 \% \mathrm{Sb}$ gegen $75 \% \mathrm{Sb}-25 \% \mathrm{Cd}$ (siehe Tab. 1) als Thermoelement unter den günstigsten konstruktiven Bedingungen zwischen $300^{\circ} \mathrm{K}$ und $270^{\circ} \mathrm{K}$ arbeiten, dann könnte man die vom Thermoelement pro Sekunde aufgenommene Wärme mit einem Nutzeffekt von höchstens

$$
\bar{\eta}_{\max }=\bar{G}_{\max }\left(T_{1}-T_{0}\right) / T_{1}=0,5 \%
$$

in entnehmbare elektrische Leistung umwandeln ${ }^{25}$.

Die mit der Thermonadel erreichbaren praktischen Gütefaktoren und Nutzeffekte sind sehr viel kleiner als die hier errechneten maximalen Werte. Dies liegt im wesentlichen an folgenden drei Tatsachen:

1. Der Formfaktor $F=1+W / W_{\mathrm{n}}$ ist bei der Thermonadel stets größer als 1 wegen der zusätzlichen Wärmeableitung durch die Trägernadel.

2. Eine „ideale Anpassung“ eines Meßgerätes an den elektrischen Widerstand der Thermonadelspitze läßt sich nicht erreichen, weil der elektrische Wider-

25 G. P. Burn s${ }^{25}$ a hat den Nutzeffekt eines Thermoelementes zwischen $427^{\circ} \mathrm{C}$ und $27^{\circ} \mathrm{C}$ zu $33,9^{\circ} \%$ berechnet, wobei er eine differentielle Thermokraft von $1000 \mu \mathrm{V} /{ }^{\circ}$ annahm. Dieses Thermoelement müßte nach unserer Berechnung jedoch einen Arbeitsfaktor $A=14,5$ besitzen. - Mit den günstigsten heute bekannten Thermopaaren $(A \approx 0,4)$ ließen sich zwischen den von Burns angenommenen Temperaturen stand der Zuleitungen zum Meßgerät nicht (wie bei den bisherigen Betrachtungen) vernachlässigt werden darf. "Zuleitungen“ mit relativ hohem elektrischem Widerstand sind die leitenden Schichten entlang der Thermonadel außerhalb des Temperaturgefälles an ihrer äußersten Spitze. Ihr elektrischer Widerstand $R_{\mathrm{z}}$ kann (abhängig vom Spitzendurchmesser der Thermonadel) wesentlich größer als der elektrische Widerstand $R$ werden. ( $R$ ist ja nur der elektrische Widerstand der Thermonadelspitze, die sich noch im Temperaturgefälle befindet.) Der elektrische Gesamtwiderstand $R_{\text {ges }}$ des Thermonadelkreises setzt sich deshalb immer aus drei Anteilen zusammen:

$$
\begin{gathered}
R_{\text {ges }}=R+R_{\mathrm{a}}+R_{\mathrm{z}} . \\
R_{\mathrm{a}}=\left(R+R_{\mathrm{z}}\right) a, \\
R_{\mathrm{z}}=b R, \\
R_{\text {ges }}=R(a+1)(b+1),
\end{gathered}
$$$$
\text { Ist etwa } \quad R_{\mathrm{a}}=\left(R+R_{\mathrm{z}}\right) a,
$$

dann wird der praktische Gütefaktor $\bar{G}_{\mathrm{T}}$ einer „richtig gebauten" Thermonadel $(n=\sqrt{p})$

$$
\bar{G}_{\mathrm{T}}=\frac{A}{A+F(a+1)(b+1)} \frac{a}{a+1} .
$$

Für $\quad a=\sqrt{[A+F(b+1)] / F(b+1)}$ wird $\bar{G}_{\mathrm{T}}$ ein Maximum.

3. Die differentiellen Thermokräfte $\varepsilon$ sind bei dünnen Schichten kleiner als bei Material in kompaktem Zustand. $\varepsilon$ erreicht bei der Thermonadel im allgemeinen nur etwa $50 \%$ der Werte des kompakten Materials. Schließlich ist auch die spezifische elektrische Leitfähigkeit von dünnen Schichten kleiner als die des kompakten Materials. Es ist nicht bekannt, ob die spezifische Wärmeleitfähigkeit dünner Schichten in demselben Maße mit der Schichtdicke abnimmt bzw. ob die Lorenz-Zahlen für dünne Schichten die gleiche Größe haben, wie für kompaktes Material.

Berücksichtigt man diese drei Tatsachen, dann er. rechnet sich für den stationären Zustand der maxi. male praktische Gütefaktor einer $\mathrm{Fe}-\mathrm{Ni}$-Thermonadel in Abhängigkeit vom Öffnungswinkel $\alpha$ der Kegelspitze mit $d$ als Parameter wie in Abb. 12

nach unserer Rechnung bestenfalls praktische Nutzeffekte von etwa $4,5 \%$ erreichen. T e l k e s${ }^{21}$ errechnet für das Thermopaar Chromnickel-Konstantan $(A=0,019)$ zwischen $530^{\circ} \mathrm{C}$ und $20^{\circ} \mathrm{C}$ einen Nutzeffekt von knapp $1 \%$, was nach unserer Rechnung auch noch zu optimistisch ist.

25a G. P. B urn s, Phys. Rev. (2) 78, 327 [1950]. 


\begin{tabular}{|c|c|c|c|c|c|c|c|c|c|c|}
\hline $\begin{array}{l}\text { Lfd. } \\
\text { Nr. }\end{array}$ & I & II & $\begin{array}{c}\varepsilon \mathrm{I} \\
\text { gegen Pt } \\
{\left[\mu \mathrm{V} /{ }^{\circ}\right]}\end{array}$ & $\begin{array}{c}\varepsilon \mathrm{II} \\
\text { gegen } \mathrm{Pt} \\
{\left[\mu \mathrm{V} /{ }^{\circ}\right]}\end{array}$ & $\begin{array}{c}L_{1} \\
{\left[\mathrm{~V}^{2} / \mathrm{O}^{2} \cdot 10^{8}\right.}\end{array}$ & $\begin{array}{c}L_{11} \\
{\left[\mathrm{~V}^{2} / 0^{2} \cdot 10^{8}\right.}\end{array}$ & $A$ & G & $G_{\max }$ & $\begin{array}{l}Q_{\mathrm{v}} \cdot\left(T_{1}-T_{0}\right) \\
\quad\left[\mathrm{cal} \cdot \mathrm{O}_{\mathrm{j}}\right.\end{array}$ \\
\hline $\left.1^{\prime}\right)$ & $\begin{array}{l}\text { PbS (mit Pb- } \\
\text { Úberschuß) }\end{array}$ & $\begin{array}{l}\text { ZnSb (mit Spuren } \\
\text { v. } \mathbf{A g} \text { u. Sn) }\end{array}$ & -100 & +230 & 2,5 & 13,8 & 0,42 & 0,29 & 0,087 & $1,4 \cdot 10^{-17}$ \\
\hline $\left.2^{2}\right)$ & $90 \% \mathrm{Bi}-10 \% \mathrm{Sb}$ & $98,5 \% \mathrm{Te}-1,5 \% \mathrm{~s}$ & -78 & +575 & 2,83 & $1750-3500$ & $0,0225-0,0115$ & $0,022-0,011$ & $0,005-0,003$ & $2,1 \cdot 10^{-16}-4,0 \cdot 10^{-16}$ \\
\hline 3 & $90 \% \mathrm{Bi}-10 \% \mathrm{Sb}$ & $\mathrm{Si}$ & -78 & +450 & 2,83 & 17000 & 0,016 & 0,014 & 0,0037 & $3,2 \cdot 10^{-16}$ \\
\hline $\left.4^{3}\right)$ & $90 \% \mathrm{Bi}-10 \% \mathrm{Sb}$ & Te Einkrist. ll & -78 & +436 & 2,83 & 175 & 0,12 & 0,10 & 0,025 & $4,7 \cdot 10^{-17}$ \\
\hline $\left.5^{3}\right)$ & $90 \% \mathrm{Bi}-10 \% \mathrm{Sb}$ & Te polykrist. & -78 & +376 & 2,83 & 150 & 0,10 & 0,096 & 0,023 & $5,1 \cdot 10^{-17}$ \\
\hline $\left.6^{4}\right)$ & $90 \% \mathrm{Bi}-10 \% \mathrm{sb}$ & $99,6 \% \mathrm{Te}-0,4 \% \mathrm{Bi}$ & -78 & +191 & 2,83 & $19-31$ & $0,19-0,12$ & $0,16-0,11$ & $0,038-0,027$ & $3,1 \cdot 10^{-17}-4,4 \cdot 10^{-17}$ \\
\hline $\left.7^{4}\right)$ & $90 \% \mathrm{Bi}-10 \% \mathrm{Sb}$ & $99,1 \% \mathrm{Te}-0,9 \% \mathrm{Sb}$ & -78 & +139 & 2,83 & $15-25$ & $0,15-0,10$ & $0,13-0,095$ & $0,031-0,026$ & $3,8 \cdot 10^{-17}-45 \cdot 10^{-17}$ \\
\hline $\left.8^{5}\right)$ & $90 \% \mathrm{Bi}-10 \% \mathrm{Sb}$ & $75 \% \mathrm{Sb}-25 \% \mathrm{Cd}$ & -78 & +112 & 2,83 & $3,4-13,8$ & $0,29-0,12$ & $0,22-0,11$ & $0,051-0,027$ & $2,3 \cdot 10^{-17}-4,4 \cdot 10^{-17}$ \\
\hline 9 & $90 \% \mathrm{Bi}-10 \% \mathrm{Sb}$ & $\mathrm{Sb}$ & -78 & +47 & 2,83 & 3,22 & 0,128 & 0,113 & 0,031 & $3,8 \cdot 10^{-17}$ \\
\hline 10 & $97 \% \mathrm{Bi}-3 \% \mathrm{Sb}$ & $\mathrm{Sb}$ & --75 & +47 & 4,06 & 3,22 & 0,102 & 0,093 & 0,022 & $5,3 \cdot 10^{-17}$ \\
\hline 11 & $\mathrm{Bi}$ & $\mathrm{Sb}$ & -70 & +47 & 3,33 & 3,22 & 0,104 & 0,094 & 0,023 & $5,1 \cdot 10^{-17}$ \\
\hline 12 & Konstantan & Chromnickel & -34 & +22 & 3.77 & 4,6 & 0,019 & 0,018 & 0,0046 & $2,6 \cdot 10^{-16}$ \\
\hline 13 & Konstantan & $\mathrm{Fe}$ & -34 & +18 & 3,77 & 3,06 & 0,020 & 0,019 & 0,0048 & $2,4 \cdot 10^{-16}$ \\
\hline 14 & Konstantan & $\mathrm{Cu}$ & -34 & $+\quad 7,5$ & 3,77 & 2,87 & 0,013 & 0,012 & 0,0032 & $3,7 \cdot 10^{-16}$ \\
\hline 15 & Konstantan & Manganin & -34 & $+\quad 6,0$ & 3,77 & 3,13 & 0,012 & 0,011 & 0,0028 & $4,2 \cdot 10^{-16}$ \\
\hline 16 & Co & $\mathrm{Fe}$ & -16 & +18 & 1,13 & 3,06 & 0,015 & 0,014 & 0,0036 & $3,3 \cdot 10^{-16}$ \\
\hline 17 & Co & $\mathrm{Rh}$ & -16 & $+\quad 6,5$ & 1,13 & 1,20 & 0,011 & 0,010 & 0,0027 & $4,4 \cdot 10^{-16}$ \\
\hline 18 & Co & $\mathrm{Ta}$ & -16 & $+\quad 5,0$ & 1,13 & 2,25 & 0,0067 & 0,0067 & 0,0017 & $7,1 \cdot 10^{-16}$ \\
\hline 19 & $\mathrm{Ni}$ & $\mathrm{Fe}$ & -15 & +18 & 1,69 & 3,06 & 0,012 & 0,011 & 0,0029 & $4,1 \cdot 10^{-16}$ \\
\hline 20 & $P d$ & $\mathrm{Fe}$ & -3 & +18 & 2,13 & 3,06 & 0,0043 & 0,0043 & 0,0011 & $1,1 \cdot 10^{-16}$ \\
\hline 21 & $P d$ & Au & -3 & +7 & 2,13 & 2,91 & 0,0010 & 0,0010 & 0,00025 & $4,7 \cdot 10^{-15}$ \\
\hline 22 & $\mathrm{Pt}$ & $\mathrm{Au}$ & 0 & +7 & 3,11 & 2,91 & 0,00041 & 0,00041 & 0,0001 & $1,2 \cdot 10^{-14}$ \\
\hline 23 & $\mathrm{Pt}$ & $90 \% \mathrm{Pt}-10 \% \mathrm{Rh}$ & 0 & $+\quad 6$ & 3,11 & 2,01 & 0,00035 & 0,00035 & 0,00009 & $1,3 \cdot 10^{-14}$ \\
\hline 24 & \multicolumn{2}{|c|}{ „Ideales Thermoelement“ } & & & & & 1,00 & 0,50 & 0,$172 ;(0,5)$ & $7,0 \cdot 10^{-18} ;\left(2,4 \cdot 10^{-18}\right)$ \\
\hline
\end{tabular}

Tab. 1.

1) M. T e 1 k e s, J. Appl. Phys. 18, 1126 [1947]. 2) A. Pe trika in u. K. J a c oby, Z. anorg. allg. Chem. 210, 195 [1933]. C a rtwrigh t, Ann. Phys., Paris 18, 656 [1933]. 
angegeben. Es ist angenommen, daß $R$ auf einer Länge $100 d$ definiert ist, und daß das Verhältnis der Dicke der maximalen Aufdampfschichten zu den Spitzendurchmessern $D_{\mathrm{m}} / d=1 / 10$ ist. Der Arbeitsfaktor für die $\mathrm{Fe}-\mathrm{Ni}$-Thermonadel $\left(A=5 \cdot 10^{-3}\right)$ ist mit Berücksichtigung von Punkt 3. unter der Annahme gleichbleibender Lorenz-Zahlen ${ }^{26}$ errechnet. Außerdem ist $a \approx 1$ und in hinreichender Annäherung an die Erfahrung $b \approx 0,2 \cdot d^{-1 / 3}$ gesetzt, wobei also für einen Spitzendurchmesser $d=10^{-5} \mathrm{~cm}$ das Verhältnis $R_{\mathrm{z}} / R=b \approx 10$ ist. (Dies bedeutet, daß bei diesem Durchmesser etwa $90 \%$ des elektrischen Gesamtwiderstandes der Thermonadel als „Zuleitungen “ angesehen werden müssen.)

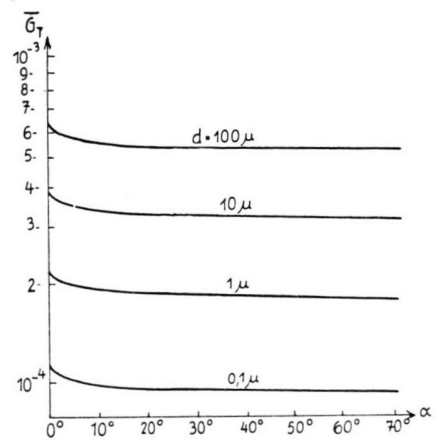

Abb. 12. Praktischer Gütefaktor $\bar{G}_{\mathrm{T}}$ einer $\mathrm{Fe}-$ Ni-Thermonadel in Abhängigkeit vom Öffnungswinkel $\alpha$ der Kegelspitze mit dem Spitzendurchmesser $d$ als Parameter.

Bisher wurde nur der stationäre Zustand an der Thermonadel betrachtet. Abb. 13 gibt den praktischen Gütefaktor einer Thermonadel im nichtstatio-

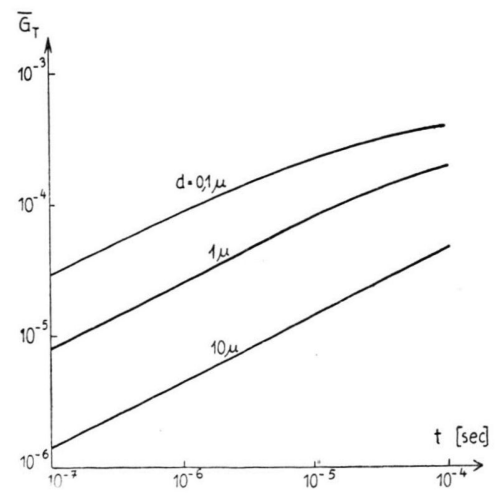

Abb. 13. Praktischer Gütefaktor $\bar{G}_{\mathrm{T}}$ einer $\mathrm{Fe}-\mathrm{Ni}$-Thermonadel in Abhängigkeit von der Meßdauer $t$ mit dem Spitzendurchmesser $d$ als Parameter. Es ist $a=1, F=2$ und $R / W=$ const angenommen.

${ }^{26}$ E. Justi, M. Kohler u. G. Lautz, Z. Naturforschg. 6a, 544 [1951].

27 M. C z e r n y, Ann. Phys., Lpz. 12, 993 [1932].

28 C. H. C a r twrig h t, Z. Phys. 92, 153 [1934]. nären Zustand in Abhängigkeit von der Meßzeit $t$ mit dem Spitzendurchmesser $d$ als Parameter wieder. Es wurde in dieser Darstellung angenommen, daß das Verhältnis $R / W$ immer praktisch konstant ist. Die jetzt zeitabhängigen Werte von $b$ in Gl. (36) wurden aus Abb. 5 entnommen, wobei $\left(T_{1}-T_{0}\right) / 20$ praktisch gleich Null gesetzt wurde. Außerdem ist $a=1$ und $F=2$.

\section{Das Thermoelement als Meßgerät}

Verwendet man ein Thermoelement als Thermometer, dann wird ein Teil der im Thermoelement erzeugten elektrischen Energie einem elektrischen Meßgerät (Galvanometer, Verstärker) zugeführt, welches diese Energie zur Anzeige verbraucht. Alle diese Meßgeräte haben jedoch, wie Czerny ${ }^{27}$, $\mathrm{C}$ a r twright ${ }^{28}$, I s ing ${ }^{29}$, Z e rnike ${ }^{30}$ u. a. gezeigt haben, eine natürliche Empfindlichkeitsgrenze. Diese wird unabhängig von der speziellen Konstruktion des Meßgerätes allein durch die statistischen Schwankungen der molekularen Wärmebewegung bestimmt. Damit das Gerät überhaupt ,anzeigen“ kann, muß die vom Meßgerät in der verfügbaren Zeit $t$ aufgenommene Energie mindestens ebenso groß sein wie der Boltzmannsche Energiebetrag ${ }^{31}$ $k T_{1}$. Dabei ist $k=1,38 \cdot 10^{-23} \mathrm{Ws} /{ }^{\circ}$ die BoltzmannKonstante und $T_{1}$ die absolute Temperatur des Meßkreises. Bei einem aperiodischen Galvanometer z. B. ist die verfügbare Zeit die Einstellzeit $t$, und bei einem Verstärker ist sie die Einschwingzeit der Schwingungskreise. Diese wird durch die Dämpfung bestimmt, also den Frequenzbereich $\Delta v$, auf den der Verstärker anspricht. Diese sogenannte Bandbreite $\Delta v$ ist deshalb

$$
\Delta v \approx 1 / t .
$$

Hat z. B. das drehbare System eines Galvanometers seinen Endausschlag erreicht, so ist der Spule während der Einstellzeit $t$ die Energie $I^{2} R_{\mathrm{a}} t$ zugeführt worden. ( $I=$ Strom, $R_{\mathrm{a}}=$ Spulenwiderstand.) Diese Energie wird größtenteils, in Wärme verwandelt. Außerdem wird aber die potentielle Energie des drehbaren Systems vergrößert: seine Feder wird gespannt. Die dazu erforderliche Spannarbeit $S$ ist

$$
S=\delta I^{2} R_{\mathrm{a}} t .
$$

${ }^{29}$ G. I s in g, Ann. Phys., Lpz. 8, 905 [1931].

30 F. Z ernike, Z. Phys. 40, 628 [1926].

31 R. W. P o h l, Einf. in die Elektrizitätslehre, 1949, 272. 
Die Zahl $\delta \leqq 1$ ist der Wirkungsgrad des Galvanometers. Durch die Brownsche Molekularbewegung hat das drehbare System (als linearer Oszillator nach dem Gleichverteilungssatz) im Mittel die Gesamtenergie $k T_{1}$. Damit eine zugeführte Energie $E$ überhaupt meßbar wird, ist es nötig, daß die Spannarbeit $S_{1}$ mindestens so groß ist wie die mittlere potentielle Energie ${ }^{\frac{1}{2}} k T_{1}$ des Systems. Das heißt, $\mathrm{da}$ die kleinste noch mit einem aperiodischen Galvanometer meßbare elektrische Energie $E_{\min }=I^{2} R_{\mathrm{a}} t$ die Größe haben muß:

$$
E_{\min } \geqq \frac{1}{\delta} \frac{1}{2} k T_{1} .
$$

Soll eine Energie so genau meßbar sein, daß der mittlere relative Fehler einer Einzelmessung nur $c^{0} \%$ beträgt, dann muß die dem Galvanometer zuzuführende Meßenergie $E_{\mathrm{G}}$ sogar die Größe haben ${ }^{32}$ :

$$
E_{\mathrm{G}} \geqq \frac{100}{c \delta} \frac{1}{2} k T_{1} \text {. }
$$

Ganz ähnlich liegen die Verhältnisse. wenn man einen Verstärker als Meßinstrument benutzt. In diesem Falle ist die kleinste noch meßbare elektrische Energie durch die verschiedenen Rauscheffekte ${ }^{34}$ in der Meßanordnung begrenzt. Betrachtet man den speziellen und günstigsten Fall, daß die frequenzabhängigen Rauscheffekte gegenüber dem „weißen“ Rauschen vernachlässigbar sind - wie z. B. bei einem von $\mathrm{Kr}$ o ebel${ }^{35}$ angegebenen Gleichspannungsverstärker - , dann kann man das Rauschen im Eingangskreis mit dem Realteil des elektrischen Widerstandes $R_{\text {ges }}$ darstellen durch das Ersatzbild eines Generators mit dem inneren Widerstand $R_{\mathrm{i}}=$ $R_{\text {ges }}$. Das Quadrat $\overline{u^{2}}$ des Effektivwertes der EMK dieses Generators beträgt nach Johns on ${ }^{36}$ und $\mathrm{Ny} \mathrm{qu}$ is t ${ }^{37}$

$$
\overline{u^{2}}=4 k T_{1} R_{\text {ges }} \Delta v .
$$

Dabei ist $k$ wieder die Boltzmann-Konstante, $T_{1}$ die Temperatur, auf der sich der Kreis befindet, und $\Delta v$ die Bandbreite, also der Frequenzbereich, in dem gemessen wird.

Die kleinste Energie $E_{\mathrm{V}}$ eines Signals, dessen Größe man mit einem mittleren relativen Fehler von $c \%$

\footnotetext{
32 Diese Grenze besteht auch für alle Galvanometerverstärker, wie z. B. den lichtelektrischen Verstärker ${ }^{32 a}$ mit seinen konstruktiven Abarten ${ }^{33}$.

32a L. M e rz , V.D.E.-Fachb. 11, 57 [1939].

33 A. S c h a ll e r, ATM Z 634-7 Febr. 1952.

34 W. Kl e en, ETZ A 76, 209 [1955].

35 W. K r o e b e l, Z. Phys. 133, 30 [1952].
}

noch messen wollte, müßte hier die Größe haben ${ }^{38}$

$$
E_{\mathrm{V}}=\frac{400}{c} k T_{1} .
$$

Die Energien $E_{\mathrm{G}}$ und $E_{\mathrm{V}}$ mögen nun von einem Thermoelement geliefert werden, und es soll im folgenden nach den Wärmemengen $Q_{\mathrm{G}}$ und $Q_{\mathrm{V}}$ gefragt werden, die man dem Thermoelement zuführen muß, damit es die elektrischen Energien $E_{\mathrm{G}}$ und $E_{\mathrm{V}}$ an ein Galvanometer und an einen Verstärker abgeben kann. Wandelt das Thermoelement Wärmeenergie mit einem praktischen Nutzeffekt $\bar{\eta}=\bar{G}\left(T_{1}-T_{0}\right) / T_{1}$ in entnehmbare elektrische Energie um, dann betragen die zur Erzeugung von $E_{\mathrm{G}}$ und $E_{\mathrm{V}}$ benötigten Wärmemengen:

$$
\begin{aligned}
Q_{\mathrm{G}} & =\frac{100}{\eta \delta c} \frac{1}{2} k T_{1}, \\
Q_{\mathrm{V}} & =\frac{400}{\eta c} k T_{1} .
\end{aligned}
$$

Dafür kann man auch schreiben:

$$
\begin{aligned}
& Q_{\mathrm{G}}\left(T_{1}-T_{0}\right)=\frac{100}{\bar{G} \delta c} \frac{1}{2} k T_{1}{ }^{2}, \\
& Q_{\mathrm{V}}\left(T_{1}-T_{0}\right)=\frac{400}{\bar{G} c} k T_{1}{ }^{2} .
\end{aligned}
$$

In den Gln. (45) und (46) ist $T_{1}-T_{0}$ die Temperaturdifferenz, die einerseits nötig war, um die thermoelektrische Spannung $\left(T_{1}-T_{0}\right)$ und damit die elektrische Energie im Thermoelement zu erzeugen, die aber andererseits auch dafür verantwortlich ist, daß das Thermoelement die Wärmemenge $Q_{\mathrm{G}}$ bzw. $Q_{\mathrm{V}}$ aufgenommen, also verbraucht hat. Betrachtet man diese Anordnungen als Thermometer, dann geben die Gln. (45) und (46) Auskunft über die Wärmemengen, die für die Messung einer Temperaturdifferenz $T_{1}-T_{0}$ mit Hilfe eines Galvanometers und eines Verstärkers mindestens benötigt werden. Diese Wärmemengen sind um so größer je kleiner die zu messende Temperaturdifferenz ist.

In Tab. 1 sind für einige Thermopaare neben der differentiellen Thermokraft $\varepsilon$, den Lorenz-Zahlen $L_{\mathrm{I}}$ und $L_{\mathrm{II}}$, dem Arbeitsfaktor $A$, den Gütefaktoren $G$ und $\bar{G}_{\max }$ auch die Minimalwerte des Produktes $Q_{\mathrm{V}}\left(T_{1}-T_{0}\right)_{\min }$ für einen Meßfehler $c=100 \% \mathrm{zu}$ -

36 J. B. J o h n s o n, Phys. Rev. 32, 97 [1928].

37 H. Ny q u is t, Phys. Rev. 32, 110 [1928].

38 Ein Vergleich der Gln. (40) und (42) zeigt, daß für $\delta=0,125$ (ein Wert, der von guten Galvanometern erreicht wird) die kleinsten Energien, die man mit einem mittleren Fehler von $c^{\%} \%$ mit einem Galvanometer und mit einem Verstärker messen kann, gleich werden. 
sammengestellt. Alle Zahlenangaben beziehen sich auf $300^{\circ} \mathrm{K}\left(T_{1} \approx T_{0}\right)$ und können vor allem bei den Legierungen keinen Anspruch auf große Zuverlässigkeit erheben. Da die elektrischen und thermischen Daten stark vom Reinheitsgrad abhängen, der nicht bekannt war (die Werte für $\varepsilon$ und $L$ wurden nur übernommen), sollen die Angaben der Tab. 1 hauptsächlich einen Vergleich verschiedener Thermopaare ermöglichen. Man sieht, daß die besten heute bekannten Thermopaare (Nr. 1) in ihrem praktischen Nutzeffekt nur noch um den Faktor $1 / 2$ hinter dem „idealen Thermoelement" zurückbleiben.

Es ist interessant, Gl. (45) einmal unter den äußersten Grenzbedingungen zu betrachten. Läßt man $c \rightarrow 100$ (völlig ungenaue Messung), $\delta \rightarrow 1$ (Verwendung eines „idealen Meßgerätes“, das auch keine thermische Unruhe des Rahmens haben soll - etwa als Folge einer Umgebungstemperatur nahe dem abs. Nullpunkt -) und $G \rightarrow 1 / 2$ (Verwendung eines Thermoelementes mit dem praktisch höchsten erzielbaren Nutzeffekt) gehen, dann sieht man leicht ein, daß noch durch das Johnson-Rauschen im Thermoelement eine untere Grenze für das Produkt $Q_{\mathrm{G}}\left(T_{1}-T_{0}\right)$ gesetzt ist. Zerlegt man etwa den elektrischen Widerstand $R$ unseres idealen Thermoelementes in die Teilwiderstände

$$
R=R_{1}+R_{2}+R_{3}+\ldots+R_{n},
$$

die sich auf den Temperaturen $T_{1} ; T_{2} ; T_{3} ; \ldots ; T_{n}$ befinden, dann liefert der Teilwiderstand $R_{n}$ zur Rauschspannung $\bar{u}^{2}$ im Thermoelement den Beitrag $u_{n}{ }^{2}=4 k \Delta v R_{n} T_{n}$ und es ist

$$
\bar{u}^{2}=4 k \Delta v \cdot \sum_{1}^{n} R_{n} T_{n}=4 k \Delta v R \frac{T_{1}+T_{0}}{2}
$$

im Falle des linearen Temperaturverlaufes in unserem Thermoelement. Die kleinste meßbare Wärmemenge wird damit ${ }^{39}$

$$
Q_{\mathrm{G}}=\frac{4 k}{\bar{\eta}} \frac{T_{1}+T_{0}}{2}=\frac{4 k}{\frac{1}{2} \frac{T_{1}-T_{0}}{T_{1}}} \cdot \frac{T_{1}+T_{0}}{2}
$$

oder

$$
Q_{G}\left(T_{1}-T_{0}\right) \geqq 4 k T_{1}\left(T_{1}+T_{0}\right) .
$$

Aus diesem Grenzübergang ersieht man, daß das Produkt aus einer beliebigen Wärmemenge $Q$ und der mit ihr ursächlich verknüpften Temperaturdifferenz $T_{1}-T_{0}$ nicht kleiner als $4 k T_{1}{ }^{2}$ gemessen

39 Man erhält das gleiche Ergebnis wie bei der Betrachtung des gleichen Grenzfalles mit einem „idealen Verstärker“ als Meßgerät. werden kann. Betrachtet man die Wärmemenge $Q_{\mathrm{G}}$ in Gl. (47) als den gesamten Wärmeinhalt des thermodynamischen Systems zwischen $T_{1}$ und $T_{0}$, dann gilt [für das Gleichheitszeichen in Gl. (47)] mit $C=$ Wärmekapazität des Systems

$$
Q_{\mathrm{G}}=C\left(T_{1}-T_{0}\right)=C \cdot \Delta T .
$$

Durch eine Messung von $T_{1}-T_{0}$ wird diesem System gerade eine solche Wärmemenge entzogen, daß die Anfangstemperatur $T_{1}$ auf $T_{0}$ absinkt. Die Temperatur des Systems wird also durch die Messung um den Betrag $T_{1}-T_{0}$ "gestört". Für dieses spezielle thermodynamische System kann man schreiben:

$$
C\left(T_{1}-T_{0}\right)^{2}=4 k T_{1}\left(T_{1}+T_{0}\right) .
$$

Setzt man für $C$ ein ganzzahliges Vielfaches der Wärmekapazität $4 k$ ein,

$$
C=N \cdot 4 k,
$$

dann kann man aus Gl. (49) $T_{0}$ und damit auch die offenbar kleinste Temperaturdifferenz

$$
T_{1}-T_{0}=\left(T_{1}-T_{0}\right)_{\min }
$$

berechnen, die in einem beliebigen thermodynamischen System der Wärmekapazität $N \cdot 4 k$ meßtechnisch noch als Grenzwert einen Sinn hat.

$$
\left(T_{1}-T_{0}\right)_{\min }=T_{1} \cdot \frac{\sqrt{8 N+1}-1}{2 N} .
$$

Um diesen Betrag muß jede Temperaturangabe eines Systems immer mindestens ungenau bleiben. Diese Ungenauigkeit ist prinzipiell nicht zu beseitigen. Man kann sie zwar durch die „Störung“ infolge des Meßvorganges entstanden denken, aber eine nachträgliche Berechnung dieser Störung ist illusorisch, weil dazu wenigstens eine Temperatur des Systems "genau“ bekannt sein müßte. Da diese „genaue" Kenntnis aber auch nur aus einer Messung stammen kann, entfällt die Voraussetzung für jede nachträgliche Korrektur. - Für $N=1$ ist die Ungenauigkeit der Temperatur vollständig, aber bereits für ein System von z. B. 72 Molekülen eines zweiatomigen Gases (bei Zimmertemperatur) ist jede Temperaturangabe nur noch mit einer prinziziellen Ungenauigkeit von $20 \%$ behaftet.

Als Folge der Ungenauigkeit der Temperatur eines thermodynamischen Systems ist aber auch der potentielle Wärmeinhalt

$$
\int_{0}^{T} C \mathrm{~d} T=N \cdot 4 k \cdot T
$$


des Systems nach Gl. (48) stets um den Betrag $C \cdot \Delta T$ unsicher. Man könnte deshalb Gl. (47) auch folgendermaßen interpretieren: Es ist unmöglich, die Temperatur und den Wärmeinhalt eines beliebigen thermodynamischen Systems so genau anzugeben, daß das Produkt aus der Ungenauigkeit der Temperatur und der Ungenauigkeit des Wärmeinhaltes kleiner als $4 k T^{2}$ wird.

Die praktischen Grenzen für die Messung von kleinen Temperaturdifferenzen unter Verwendung von Thermoelementen liegen weit oberhalb der durch Gl. (47) gegebenen theoretischen Grenze.

Aus den Gln. (45) und (46) erkennt man, daß bei einer absoluten Temperatur $T_{1}$ der Meßstelle eines Thermoelementes um so kleinere Temperaturdifferenzen $T_{1}-T_{0}$ noch meßbar sein werden, je größer die Wärmeaufnahme eines Thermoelementes pro Grad und Sekunde (bei konstantem Nutzeffekt) und je größer die Meßzeit $t$ ist. (Die Wärmeaufnahme ist unter diesen Voraussetzungen nur ahhängig von der konstruktiven Bemessung des Thermoelementes.) Umgekehrt bedeutet dies, daß für Thermoelemente mit besonders geringem Wärmeverbrauch, z. B. für die Thermonadel, eine auch schon für die Praxis zu beachtende kleinste noch meßbare Temperaturdifferenz $\left(T_{1}-T_{0}\right)_{\min }$ existieren muß. $\left(T_{1}-T_{0}\right)_{\min }$ läßt sich mit Hilfe der Beziehungen (27), (12) und (29) aus

$$
\begin{aligned}
Q\left(T_{1}-T_{0}\right) & =\left(\pi_{1} I+j F\right)\left(T_{1}-T_{0}\right) t \\
& =\left(\frac{\varepsilon^{2}\left(T_{1}-T_{0}\right) T_{1}}{R_{\operatorname{ges}}}+\frac{T_{1}-T_{0}}{W} F\right)\left(T_{1}-T_{0}\right) t
\end{aligned}
$$

unter Beachtung von (21), (22), (36) und

$$
\bar{G}_{\mathrm{T}}=\frac{\varepsilon^{2}}{\varepsilon^{2}+\left(R / W T_{1}\right) \cdot(a+1)(b+1) F} \frac{a}{a+1}
$$

berechnen. Es ist:

$$
\left(T_{1}-T_{0}\right)_{\min }=\frac{10}{\varepsilon} \sqrt{\frac{R_{\text {ges }}}{c \delta t} \frac{a+1}{a} \frac{1}{2} k T_{1}} .
$$

Gl. (53) gilt für ein Galvanometer mit der aperiodischen Einstellzeit $t$. Für einen Verstärker mit der Bandbreite $\Delta v$ kann man ganz entsprechend schreiben:

$$
\left(T_{1}-T_{0}\right)_{\min }=\frac{20}{\varepsilon} \sqrt{\frac{R_{\text {ges }}}{c} \frac{a+1}{a} k T_{1} \Delta v} .
$$

Abb. 14 gibt $\left(T_{1}-T_{0}\right)_{\text {min }}$ als Funktion von $R_{\text {ges }}$ eines thermoelektrischen Meßkreises mit $\varepsilon$ als Parameter wieder. Die Meßdauer $t$ beträgt $\frac{1}{2} \mathrm{~s}$, der zugelassene Meßfehler $c=10 \%$, der Wirkungsgrad des verwendeten Galvanometers $\delta=0,1, a=1$ und $T_{1}=300^{\circ} \mathrm{K}$.

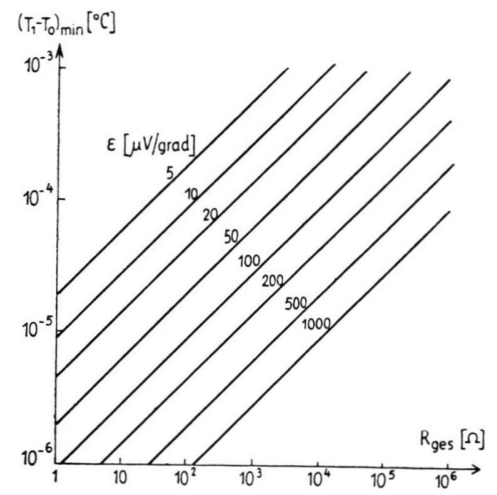

Abb. 14. Die kleinste noch meßbare Temperaturdifferenz $\left(T_{1}-T_{0}\right)$ min als Funktion des Ohmschen Widerstandes $R_{\text {ges }}$ des Meßkreises mit der differentiellen Thermospannung $\varepsilon$ des Thermoelementes als Parameter. Meßdauer $t=\frac{1}{2} \mathrm{~s}$, Meßfehler $10^{\%} \%, T_{1}=300^{\circ} \mathrm{K}$, Wirkungsgrad des verwendeten Galvanometers $\delta=0,1, a=1$.

Abb. 15 zeigt $\left(T_{1}-T_{0}\right)_{\min }$ in Abhängigkeit von der Meßdauer $t=1 / \Delta v$ für ein Thermoelement mit $\varepsilon=$ $22 \mu \mathrm{V} /^{\circ}(\mathrm{Fe}-\mathrm{Ni}$-Thermonadel $) . R_{\text {ges }}$ ist als Parameter angegeben, $a=1, c=10^{\%} \%$ und $T_{1}=300^{\circ} \mathrm{K}$. Man ersieht aus Abb. 15, daß Temperaturänderungen in kleiner werdenden Meßzeiten nur dann noch meßbar sind, wenn die Temperaturdifferenzen größer werden oder aber der Meßkreis-Widerstand abnimmt.

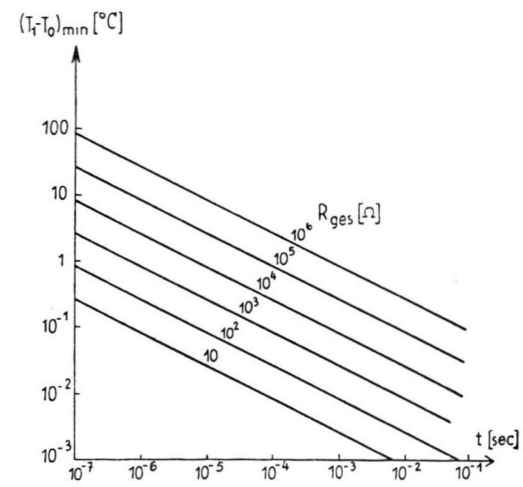

Abb. 15. $\left(T_{1}-T_{0}\right)_{\min }$ in Abhängigkeit von der Meßdauer $t=1 / \Delta v$ für ein Thermoelement mit $\varepsilon=22 \mu \mathrm{V} /{ }^{\circ}$ (z. B. Fe $-\mathrm{Ni}$ Thermonadel). Als Parameter ist $R_{\text {ges }}$ eingezeichnet $T_{1}=300^{\circ} \mathrm{K}, c=10^{\%} \%$ und $a=1$.

Damit sind die wesentlichen Größen erfaßt, die für die meßtechnische Beurteilung eines Thermoelementes im allgemeinen und der Thermonadel im speziellen benötigt werden, ohne daß bisher aber das Meßobjekt berücksichtigt wurde. 
Es bleibt nun noch die Frage zu untersuchen, in welcher Weise die Messung einer Temperaturdifferenz $T_{1}-T_{0}$ die Temperatur des Meßobjektes verändert. Alle bisher aufgestellten praktischen Beziehungen haben beim Meßvorgang nur den einen Partner, nämlich das Meßgerät, z. B. die Thermonadel berücksichtigt, der eine Wärmemenge $Q$ zugeführt wird, die nach Umwandlung in elektrische Energie gemessen wird. Woher diese Wärmemenge kommt und wie der Entzug dieser Wärmemenge sich auf das Meßobjekt auswirkt, diese Frage wurde bisher nicht betrachtet.

Jede Temperaturmessung bewirkt eine Störung des zu messenden thermischen Zustandes eines Systems, die im folgenden für einen Grenzfall diskutiert werden soll.

Die Störung des Meßobjektes ist offenbar für hinreichend kurze Meßzeiten in erster Näherung abhängig vom Verhältnis $V$ des Wärmeinhaltes $C\left(T_{1}-T_{0}\right) \quad(C=$ Wärmekapazität $)$ des Meßobjektes zur Wärmemenge $Q_{\mathrm{V}}$, die für die Messung von $T_{1}-T_{0}$ mit einem mittleren relativen Fehler von $c \%$ mindestens verbraucht wird. Mit Beziehung (46) kann man für dieses Verhältnis schreiben:

$$
V=C\left(T_{1}-T_{0}\right)^{2} \bar{G}_{\mathrm{T}} c / 400 k T_{1}{ }^{2}
$$

Für $V=100 / c$ wird die Temperaturverringerung des Meßobjektes durch den Meßvorgang ebenso groß wie der zugelassene mittlere relative Fehler $c$ der Einzelmessung von $T_{1}-T_{0}$. In diesem Falle muß die Wärmekapazität des Meßobjektes bei Verwendung eines Verstärkers als elektrisches Meßgerät die Größe haben:

$$
C_{\min }=4 \cdot 10^{4} k T_{1}^{2} / c^{2} \bar{G}_{\mathrm{T}}\left(T_{1}-T_{0}\right)^{2} .
$$

Abb. 16 gibt $C_{\text {min }}$ und den Durchmesser von Wasserkugeln der Wärmekapazität $C_{\min }$ in Abhängigkeit von $T_{1}-T_{0}$ mit dem praktischen Gütefaktor $G$ als Parameter wieder. Es ist dabei : $c=10 \%, T_{1}=300^{\circ} \mathrm{K}$.

Die Berechnung einer Störung des thermischen Zustandes in sehr kleinen Meßobjekten durch einen Meßvorgang hat in der vorliegenden Form offensichtlich nur dann einen Sinn, wenn man das Meßobjekt als ein hinreichend adiabatisches System betrachten, also die Wärmeableitung in die Umgebung des Meßobjektes während der Meßdauer vernachläs- sigen kann. Die Wärmeableitung in die Umgebung wird man um so eher vernachlässigen können, je schlechter die Umgebung Wärme leitet und je schneller die Messung vor sich geht.

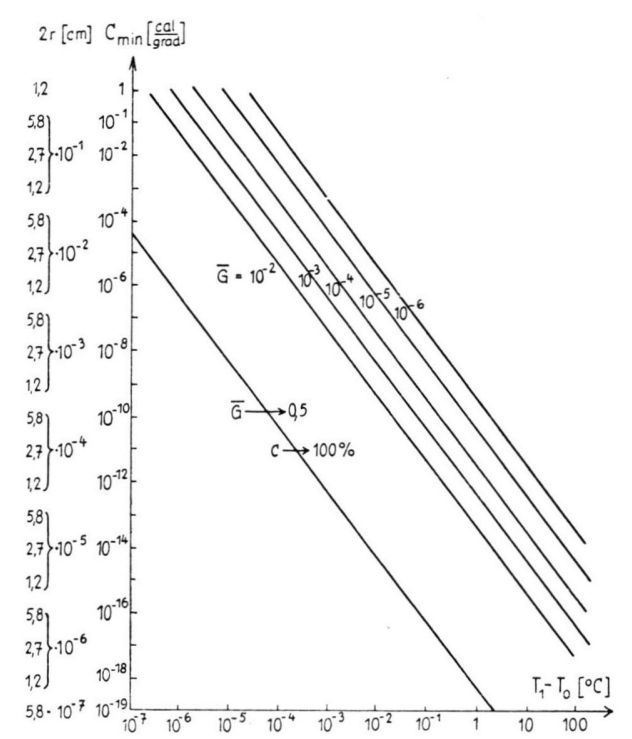

Abb. 16. Die minimale Wärmekapazität $C_{\min }$ eines Meßobjektes und der Durchmesser $2 r$ von Wasserkugeln der Wärmekapazität $C_{\min }$ als Funktion der zu messenden Temperaturdifferenz $T_{1}-T_{0}$ zwischen Meßobjekt und Umgebung mit dem praktischen Gütefaktor $\bar{G}$ des zur Messung verwendeten Thermoelementes als Parameter. Meßfehler $10^{\circ} \%, T_{1}=300^{\circ} \mathrm{K}$.

Kann man ein kleines Meßobjekt innerhalb der Meßzeit jedoch nicht mehr als ein hinreichend adiabatisches System betrachten, dann wird man es in der Praxis im wesentlichen nur noch mit zwei anderen Möglichkeiten zu tun haben: Entweder das Meßobjekt enthält während eines Zeitraumes, der groß im Verhälntis zur Meßzeit ist, eine Wärmequelle, oder dies ist nicht der Fall.

Ist eine Wärmequelle im Meßobjekt vorhanden, dann wird das Meßobjekt im allgemeinen bereits in einem thermischen Gleichgewicht mit seiner Umgebung stehen und dann ohnehin mehr Wärme an diese abgeben als die Thermonadel für die Messung verbraucht. Ist eine derartige Wärmequelle jedoch nicht vorhanden und die Meßzeit nicht kurz genug, daß man das Objekt als ein adiabatisches System betrachten kann, dann wird das zu messende "Wärmedepot" im allgemeinen wohl schon „zerflossen" sein, bevor es gemessen werden kann. 


\section{Schlußbemerkung}

Derartige Überlegungen könnten zusammen mit dem Einsatz von geeigneten Meßgeräten ${ }^{40}$, wie z. B. der Thermonadel, für eine Reihe von Fragestellungen auf den verschiedensten Gebieten von Interesse werden.

Es sei abschließend nur ein Beispiel skizziert.

Bei der Absorption von Röntgenquanten treten hauptsächlich drei Effekte als Energieverbraucher auf:

1. Der Photoeffekt, 2. Der Compton-Effekt und 3. Die Paar-Bildung. Sieht man von der Paar-Bildung ab, die nur bei relativ hohen Energien auftritt, dann kann man sagen, daß sich die von einem Röntgenquant abgegebenen Energiebeträge durch mehrfache Compton- und Photoeffekte in immer kleinere Energiebeträge aufsplittern. Am Ende dieses Prozesses steht eine große Anzahl von gebildeten Ionenpaaren, deren Erzeugung in Luft z. B. im Mittel eine Energie von $32 \mathrm{eV}$ pro Paar erfordert. Letzten Endes wird aber auch die Energie dieser Ionenpaare im wesentlichen als Wärme im absorbierenden Medium erscheinen. Da die praktischen Reichweiten von Elektronen geringer Energie in Materie nicht sehr groß sind ${ }^{41}$, wird sich die Absorption von Strahlung in Materie u. a. auch durch das Auftreten von (sicher sehr kurzzeitigen) Temperaturspitzen bemerkbar machen. Nimmt man einmal an, daß sich die Energie eines Ionenpaares mittlerer Größe $\left(1,2 \cdot 10^{-18} \mathrm{cal}\right)$ in einer Wasserkugel vom Durchmesser $10 \mathrm{~m} \mu$ (Wärmekapazität $C=5 \cdot 10^{-19} \mathrm{cal} /{ }^{\circ}$ ) vollständig in Wärme umsetzt, dann wird diese Wasserkugel um etwa $2^{\circ}$ erwärmt. Wie man aus Gl. (56) ersieht, wäre der Nachweis (Verzicht auf jede Meßgenauigkeit, d. h. $c \rightarrow 100$ ) dieser Temperaturspitze - abgesehen von allen anderen Gesichtspunkten - theoretisch nur mit einem Thermoelement möglich, dessen praktischer Gütefaktor $\bar{G}=0,48$ wäre. Da $\bar{G} \leqq 0,5$ ist, läge dieser

\footnotetext{
40 Neben der Konstruktion von Thermonadeln ist die Herstellung von unpolarisierbaren Mikroelektroden ( $\mathrm{pH}_{\mathrm{H}}$-Messung, Messung von Membran- und Diffusionspotentialen in kleinen Raumbereichen) und Mikrokondensatoren (Messung kleiner bewegter Ladungsträger durch Influenz) gelungen. Es wurden dabei ähnliche Konstruktionsprinzipien wie bei der Thermonadel angewendet.
}

Nachweis also gerade an der theoretischen Grenze der Meßbarkeit.

Geht man zu größeren Energien über, dann kann man jedoch in einen Bereich kommen, in dem ein thermischer Nachweis der Absorption von Röntgenoder z. B. auch $\alpha$-Strahlen unter gewissen Voraussetzungen experimentell nicht mehr völlig aussichtslos erscheint. Könnte man etwa in einer Wasserkugel mit einem Durchmesser von 0,5 $\mu\left(C=10^{-14}\right.$ $\mathrm{cal} /{ }^{\circ}$ ) gleichzeitig eine Energie absorbieren, die $1 \mathrm{MeV}$ entspricht $\left(3,8 \cdot 10^{-14} \mathrm{cal}\right)$, dann würde sich diese Kugel um etwa $3^{\circ}$ erwärmen. Aus Gl. (56) ersieht man, daß der praktische Gütefaktor einer für den Nachweis dieser Temperaturspitze benützten Thermonadel mindestens $\bar{G}_{\mathrm{T}}=6 \cdot 10^{-5}$ betragen müßte, wenn man einen Meßfehler von $50 \%$ zuläßt. Wie man aus Abb. 13 ersieht, wäre der Nachweis einer derartigen Temperaturspitze mit einer Eisen-Nickel-Thermonadel vom Spitzendurchmesser $d=0,2 \mu$ schon in einer Meßzeit von $\sim 10^{-6}$ s möglich, die kurz genug wäre, daß man das Meßobjekt als hinreichend adiabatisches System betrachten kann.

Neben derartigen Problemen der ortsabhängigen Energieabsorption könnte man auch an die Untersuchung von ortsabhängigen chemischen Prozessen herangehen, wie sie etwa an Katalysatoren stattfinden. Ferner könnte man auch an die Messung von Temperatursprüngen an Phasengrenzen (z. B. Gas - Flüssigkeit) mit Thermonadeln denken ${ }^{43}$. Von besonderem Interesse dürfte die Behandlung energetischer Fragestellungen in der Biologie, z. B. in der experimentellen Zytologie, werden. Temperaturund Wärmemessungen mit Hilfe von Thermonadeln könnten hier vielleicht zusammen mit der Messung von Aktionspotentialen und der Bestimmung von $\mathrm{p}_{\mathrm{H}}$-Werten innerhalb der lebenden Zelle einen Beitrag zum Problem der Zuordnung von Struktur und Funktion leisten.

Herrn Professor C z erny, Herrn Professor H un d sowie Herrn Professor D ä n z e r, Frankfurt a. M., ist der Verfasser für Hinweise und Diskussionen zu Dank verpflichtet.

${ }^{41}$ Die praktische Reichweite von 2,5-kV-Elektronen in Wasser beträgt ${ }^{42}$ etwa $0,5 \mu$.

${ }_{42}$ L a nd old Börnstein, I. Bd. Atom und Molekularphysik, 5. Teil, S. 339.

43 Derartige Temperatursprünge (von etwa $10^{\circ}$ ) wurden vom Verfasser an der Oberfläche von Wassertröpfchen schon gemessen. 